\title{
Homological Solution of the Lanczos Problems in Arbitrary Dimension
}

\author{
Jean-Francois Pommaret [ \\ CERMICS, Ecole des Ponts ParisTech, France \\ Email: jean-francois.pommaret@wanadoo.fr
}

How to cite this paper: Pommaret, J.-F. (2021) Homological Solution of the Lanczos Problems in Arbitrary Dimension. Journal of Modern Physics, 12, 829-858. https://doi.org/10.4236/jmp.2021.126053

Received: April 20, 2021

Accepted: May 14, 2021

Published: May 17, 2021

Copyright $\odot 2021$ by author(s) and Scientific Research Publishing Inc. This work is licensed under the Creative Commons Attribution International License (CC BY 4.0).

http://creativecommons.org/licenses/by/4.0/ (c) (i) Open Access

\begin{abstract}
When $\mathcal{D}$ is a linear partial differential operator of any order, a direct problem is to look for an operator $\mathcal{D}_{1}$ generating the compatibility conditions (CC) $\mathcal{D}_{1} \eta=0$ of $\mathcal{D} \xi=\eta$. Conversely, when $\mathcal{D}_{1}$ is given, an inverse problem is to look for an operator $\mathcal{D}$ such that its $\mathrm{CC}$ are generated by $\mathcal{D}_{1}$ and we shall say that $\mathcal{D}_{1}$ is parametrized by $\mathcal{D}=\mathcal{D}_{0}$. We may thus construct a differential sequence with successive operators $\mathcal{D}, \mathcal{D}_{1}, \mathcal{D}_{2}, \cdots$, each operator parametrizing the next one. Introducing the formal adjoint ad () of an operator, we have $\mathcal{D}_{i} \circ \mathcal{D}_{i-1}=0 \Rightarrow \operatorname{ad}\left(\mathcal{D}_{i-1}\right) \circ \operatorname{ad}\left(\mathcal{D}_{i}\right)=0$ but $\operatorname{ad}\left(\mathcal{D}_{i-1}\right)$ may not generate all the CC of $\operatorname{ad}\left(\mathcal{D}_{i}\right)$. When $D=K\left[d_{1}, \cdots, d_{n}\right]=K[d]$ is the (non-commutative) ring of differential operators with coefficients in a differential field $K$, then $\mathcal{D}$ gives rise by residue to a differential module $M$ over $D$ while $\operatorname{ad}(\mathcal{D})$ gives rise to a differential module $N=\operatorname{ad}(M)$ over $D$. The differential extension modules ext ${ }^{i}(M)=\operatorname{ext}_{D}^{i}(M, D)$ with ext $^{0}(M)=\operatorname{hom}_{D}(M, D)$ only depend on $M$ and are measuring the above gaps, independently of the previous differential sequence, in such a way that ext $t^{1}(N)=t(M)$ is the torsion submodule of $M$. The purpose of this paper is to compute them for certain Lie operators involved in the theory of Lie pseudogroups in arbitrary dimension $n$ and to prove for the first time that the extension modules highly depend on the Vessiot structure constants $c$. Comparing the last invited lecture published in 1962 by Lanczos with a commutative diagram that we provided in a recent paper on gravitational waves, we suddenly understood the confusion made by Lanczos between Hodge duality and differential duality. We shall prove that Lanczos was not trying to parametrize the Riemann operator but its formal adjoint Beltrami $=$ ad (Riemann) which can indeed be parametrized by the operator Lanczos =ad (Bianchi) in arbitrary dimension, "one step further on to the right" in the Killing se-
\end{abstract}


quence. Our purpose is thus to revisit the mathematical framework of Lanczos potential theory in the light of this comment, getting closer to the theory of Lie pseudogroups through double differential duality and the construction of finite length differential sequences for Lie operators. In particular, when one is dealing with a Lie group of transformations or, equivalently, when $\mathcal{D}$ is a Lie operator of finite type, we shall prove that ext $^{i}(M)=0, \forall 0 \leq i \leq n-1$. It will follow that the Riemann-Lanczos and Weyl-Lanczos problems just amount to prove such a result for $i=1,2$ and arbitrary $n$ when $\mathcal{D}$ is the classical or conformal Killing operator. We provide a description of the potentials allowing to parametrize the Riemann and the Weyl operators in arbitrary dimension, both with their adjoint operators. Most of these results are new and have been checked by means of computer algebra.

\section{Keywords}

Differential Sequence, Variational Calculus, Lanczos Potential, Lanczos Operator, Vessiot Structure Equations

\section{Introduction}

Introducing the Lanczos potential $L=\left(L_{i j, k}\right)$ as a 3-tensor satisfying the algebraic relations:

$$
L_{i j, k}+L_{j i, k}=0, L_{i j, k}+L_{j k, i}+L_{k i, j}=0
$$

Lanczos claimed to have parametrized the Riemann tensor $R$ through the relation:

$$
R_{k l, i j}=\nabla_{j} L_{k l, i}-\nabla_{i} L_{k l, j}+\nabla_{l} L_{i j, k}-\nabla_{k} L_{i j, l}
$$

where $\nabla$ is the covariant derivative. However, even if we can easily verify the algebraic conditions that must be satisfied by a Riemann candidate, namely:

$$
R_{k l, i j}=-R_{l k, i j}=-R_{k l, j i}=R_{i j, k l}, R_{k l, i j}+R_{k i, j l}+R_{k j, l i}=0, R_{i, r j}^{r}=R_{i j}=R_{j i} \neq 0
$$

the generating compatibility conditions (CC) of the underlying operator for the left member cannot be the (second) Bianchi identities.

$$
B_{l, i j r}^{k} \equiv \nabla_{r} R_{l, i j}^{k}+\nabla_{i} R_{l, j r}^{k}+\nabla_{j} R_{l, r i}^{k}=0
$$

which are produced by the well known parametrization described by the Riemann operator acting on a perturbation $\Omega \in S_{2} T^{*}$ of the background metric $\omega$, that is, when $\omega$ is the Minkowski metric:

$$
2 R_{k l, i j}=\left(d_{l i} \Omega_{k j}-d_{l j} \Omega_{k i}\right)-\left(d_{k i} \Omega_{l j}-d_{k j} \Omega_{l i}\right) \Rightarrow d_{r} R_{l, i j}^{k}+d_{i} R_{l, j r}^{k}+d_{j} R_{l, r i}^{k}=0
$$

This contradiction can also be checked directly by substitution because we have:

$$
R_{k l, i j}=d_{j} L_{k l, i}-d_{i} L_{k l, j}+d_{l} L_{i j, k}-d_{k} L_{i j, l} \Rightarrow d_{r} R_{l, i j}^{k}+d_{i} R_{l, j r}^{k}+d_{j} R_{l, r i}^{k} \neq 0
$$

Then Lanczos tried to parametrize the Weyl tensor $C$, only knowing the algebraic conditions that must be satisfied by a Weyl candidate, namely: 


$$
C_{k l, i j}=-C_{l k, i j}=-C_{k l, j i}=C_{i j, k l}, C_{k l, i j}+C_{k i, j l}+C_{k j, l i}=0, C_{i, r j}^{r}=0
$$

The last condition is reducing the number of linearly independent components from 20 to 10 for space-time, that is when the dimension is $n=4$, but the previous contradiction still holds.

50 years ago, while the author of this paper was "professional" in GR under the leadership of Prof. A. Lichnerowicz, he became familiar with the Lanczos problems. Since that time, he had no wish at all to enter this kind of "private domain" where a few persons were writing alternatively. Also, all the papers were covered with "computations" involving many technical formulas, one paper using computer algebra, another Gröbner bases, another Cartan exterior calculus, another Janet bases and so on during these 50 years. Finally, the author started to have doubts on the differential geometric conformal framework. A long time after, in 2001 and for quite different reasons, namely revisiting controllability in control theory on one side and the intrinsic proof of the impossibility to find potentials for Einstein equations in vacuum (contrary to the general dream of the GR community till now!) on the other side, the author wrote a big book, published by Kluwer (See Zbl 1079.93001). At this moment, being more familiar with differential homological algebra and the "parametrization problem", the way towards the Lanczos problems became easier and we present it in four steps:

1) In the only dimension $n=4$ considered indeed by Lanczos, the so-called Lanczos "potential" $L_{i j, k}=-L_{j i, k}$ has $6 \times 4=24$ components. As they must be related by the 4 additional relations $L_{i j, k}+L_{j k, i}+L_{k i, j}=0$, we get 20 independent components, namely the number of (second) Bianchi identities. We claim that only the knowledge of the Spencer $\delta$-cohomology allows to exhibit the proper identification with the Bianchi candidate vector bundle $F_{2}$ in the short exact sequence of vector bundles where $g_{1} \subset T^{*} \otimes T$ is the symbol of the Killing equations:

$$
0 \rightarrow F_{2} \rightarrow \wedge^{3} T^{*} \otimes g_{1} \stackrel{\delta}{\rightarrow} \wedge^{4} T^{*} \otimes T \rightarrow 0
$$

and $\operatorname{dim}\left(F_{2}\right)=24-4=20$ when $n=4$. However, speaking about "potential" also means "parametrization", ... but of what?. Here comes the main confusion of Lanczos, familiar with electromagnetism (EM) while using mainly quadratic lagrangians with the Riemann tensor in place of the EM field $F$ such that $d F=0$, with the Bianchi identities as differential constraint in the corresponding variational calculus. The operator that must be parametrized indeed by means of the formal adjoint of the Bianchi operator is thus the formal adjoint of the Riemann operator, going now backwards, that is from right to left in the adjoint sequence of the Killing resolution. Such a construction, using quite difficult results (side changing functor) of homological algebra, could not have been discovered by Lanczos and followers as such tools have only been available after 1995 through the works of pure mathematicians not interested by applications. As a byproduct, this new framework is allowing in particular to replace technical 
formulas by diagram chasing, without ANY formula.

2) As for the differential sequence involved, people use to refer to E. Calabi or H. Goldschmidt who effectively gave "ad hoc" results a long time ago, around 1965 but are not quoted because they cannot be used for our purpose. However, one should rather refer to the author's many books (In particular the first one, published in 1978 and translated by MIR, Moscow, in 1983) in order to discover that such a differential sequence can be constructed for any Lie pseudogroup by using the Vessiot structure equations, still not known after 125 years!. This sequence is much more useful than the sequence constructed by GoldschmidtSpencer who have never been aware of the work of Vessiot and has nothing to do with the work of Cartan who ignored this work.

3) Going from left to right in the differential sequence, the Riemann operator is generating the compatibility conditions (CC) of the Killing operator and the Bianchi operator is generating the $\mathrm{CC}$ of the Riemann operator. However, going backwards, that is to say from right to left, by taking the respective adjoint operators, it is not true in general that the successive operators have the same property. This remark has been the reason for introducing the differential extension modules in homological algebra, the aim being to study the possible "gaps" just described. By chance, in this case it works, contrary to what could happen in the example given of the infinite dimensional Lie pseudogroup of contact transformations. Indeed, in such a case, the differential sequence is existing because the Vessiot structure equation has only one Vessiot structure constant (totally unknown) like in the example of the constant Riemannian curvature. The case of unimodular contact transformations is even more difficult with two Vessiot structure constants but no link with any Maurer-Cartan equation.

4) Last but not least, the case of conformal isometries is even much more tricky:

First of all, it is clear that, when $n \geq 4$, the first order Killing operator must be replaced by the first order conformal Killing operator while the second order Riemann operator must be replaced by the second order Weyl operator. However, ... what operator should be used in place of the first order Bianchi operator? No chance, because we shall discover that, in dimension 4, it is a second order operator!. Such a result, neither known nor acknowledged up to now, has been checked with computer algebra by the author's former PhD student A. Quadrat (INRIA) and appeared in book form ([1]). Acordingly, there does not exist a single reference on such a result. Needless to say that, in any other smaller or higher dimension, this material could not have been known by Lanczos himself or followers, a fact justifying the initial claim on the use of the Spencer $\delta$-cohomology. For example, when $n=3$, the analogue of the Riemann operator is a third order operator with first order CC.

In order to recapitulate the above procedure, we have the following differential sequence, indicating below the fiber dimensions of the vector bundles involved with $F_{0}=S_{2} T^{*}$ : 


$$
\begin{gathered}
0 \rightarrow \Theta \rightarrow T \stackrel{\text { Killing }}{\rightarrow} F_{0} \stackrel{\text { Riemann }}{\rightarrow} F_{1} \stackrel{\text { Bianchi }}{\rightarrow} F_{2} \rightarrow \cdots \\
0 \rightarrow \Theta \rightarrow n \rightarrow n(n+1) / 2 \rightarrow n^{2}\left(n^{2}-1\right) / 12 \rightarrow n^{2}\left(n^{2}-1\right)(n-2) / 24 \rightarrow \cdots
\end{gathered}
$$

where $\Theta$ is the sheaf of Killing vector fields for the Minkowski metric. Defining the operators:

$$
\text { Cauchy }=\text { ad }(\text { Killing }), \text { Beltrami }=\text { ad }(\text { Riemann }), \text { Lanczos }=\text { ad }(\text { Bianchi })
$$

we shall prove that Lanczos was in fact dreaming to construct the adjoint differential sequence:

$$
0 \leftarrow \operatorname{ad}(T) \stackrel{\text { Cauchy }}{\leftarrow} \operatorname{ad}\left(F_{0}\right) \stackrel{\text { Beltrami }}{\leftarrow} \operatorname{ad}\left(F_{1}\right) \stackrel{\text { Lanczos }}{\leftarrow} \operatorname{ad}\left(F_{2}\right) \leftarrow \cdots
$$

where $\operatorname{ad}(E)=\wedge^{n} T^{*} \otimes E^{*}$ for any vector bundle $E$ where $E^{*}$ is obtained from $E$ by inverting the transition rules when changing local coordinates, exactly like $T$ and $T^{*}$. Accordingly, all the problem will be to prove that each operator is indeed parametrized by the preceding one. As we shall see, the conformal situation could be treated similarly while starting with the conformal Killing operator followed by the Weyl operator and replacing each classical vector bundle $F$ by the corresponding conformal bundle $\hat{F}$. However, this will lead to a true nonsense because we shall discover that the analogue of the Weyl operator is of order 3 when $n=3$ while the analogue of the Bianchi operator is of order $2, \ldots$ just when $n=4$. These striking results have been confirmed by computer algebra and the reader can even find the details in book form ([1]). It follows that both the Riemann and Weyl frameworks of the Lanczos potential theory must be entirely revisited. The aim of this paper is to overcome these problems by using differential homological algebra.

C. Lanczos (1893-1974) wrote three main papers $(1939,1949,1962)$ on the search of potentials for parametrizing the Riemann and Weyl tensors ([2] [3] [4] [5]) and we refer the reader to the nice historical survey ([6]) for more details. However, Lanczos has been invited in 1962 by Prof. A. Lichnerowicz to lecture in France and this last lecture has been published in french. Getting inspiration from what happens in electromagnetism (EM) where the geometrical first set of Maxwell equations $d F=0$ when $F \in \wedge^{2} T^{*}$ is a closed 2-form can be parametrized by $d A=F$ for an arbitrary potential $A \in T^{*}$ with standard notations (See [7] for details), Lanczos created the concept of "candidate" while noticing that the Riemann and Weyl 4-tensors must "a priori" satisfy algebraic relations reducing the number of their components $R_{k l, i j}$ and $C_{k l, i j}$ respectively to 20 and 10 when $n=4$. Now, we have proved in many books ([8]-[13]) or papers ([14] [15] [16] [17] [18]) that it is not possible to understand the mathematical structure of the Riemann and Weyl tensors, both with their splitting link, without the following four important comments:

- The results discovered by E. Vessiot as early as in 1903 ([19]) are still neither known nor even acknowledged today, though they generalize the constant Riemaniann curvature condition discovered 25 years later by L. P. Eisenhart ([20]). They also allow to understand the direct link existing separately be- 
tween the Riemann tensor and the Lie pseudogroup of isometries of a nondegenerate metric on one side or between the Weyl tensor and the group of conformal isometries of this metric on another side. Vessiot proved that, for any Lie pseudogroup $\Gamma \in \operatorname{aut}(X)$ the pseudogroup of all local diffeomorphisms, there is a geometric object $\omega$, may be of a high order $q$ large enough and not of a tensorial nature, which is characterizing $\Gamma$ in the sense that one has:

$$
\Gamma=\left\{f \in \operatorname{aut}(X) \mid \Phi_{\omega}\left(j_{q}(f)\right)=j_{q}(f)^{-1}(\omega)=\omega\right\}
$$

where $\{\Phi\}$ is a fundamental set of differential invariants of order $q$ and $\omega$ must satisfy certain (non-linear in general) integrability conditions of the form:

$$
I\left(j_{1}(\omega)\right)=c(\omega)
$$

called Vessiot structure equations, depending on a certain number of Vessiot structure constants $c$ eventually satisfying algebraic Jacobi conditions $J(c)=0$ and we let the reader compare this situation to the Riemann or contact cases ([1]). We want to point out that these structure equations were perfectly known by E. Cartan (1869-1951) who never said that these results were at least competing with or even superseding the corresponding Cartan structure equations that he has developed about at the same time for similar purposes. The underlying reason is of a purely personal origin related to the differential Galois Theory within a kind of "mathematical affair" involving the best french mathematicians of that time. The original letters, given to the author of this paper by M. Janet, a friend of E. Vessiot, have ben published in ([10]) and have been put as a deposit in the main library of Ecole Normale Supérieure in Paris for future historical studies.

- A nonlinear operator with second member does not in general admit CC, ... unless it corresponds to the defining equations in Lie form of a Lie pseudogroup and the CC are the Vessiot structure equations in that case with structure constants determined by the chosen geometric object (compare again to the Riemannian geometry). We have shown in many books already quoted that, if $\mathcal{D}$ is a Lie operator and we set $\Theta=\{\xi \in T \mid \mathcal{D} \xi=0\}$, with bracket $[\Theta, \Theta] \in \Theta$ induced by the ordinary bracket of vector fields, then the system $\mathcal{D} \xi=\Omega$ is the linearization of a non-linear version when $\Omega$ is a perturbation of $\omega$ (twice the infinitesimal deformation tensor in elasticity) along the formula:

$$
\mathcal{D} \xi=\mathcal{L}(\xi) \omega=\left.\frac{\mathrm{d}}{\mathrm{d} t}\left(j_{q}(\exp (t \xi))^{-1}(\omega)\right)\right|_{t=0}
$$

Similarly, we can choose for the generating CC $\mathcal{D}_{1}$ the linearization of a non-linear version described by the Vessiot structure equations:

$$
\frac{\partial I}{\partial j_{1}(\omega)}\left(j_{1}(\omega)\right) j_{1}(\Omega)=\frac{\partial c}{\partial \omega}(\omega) \Omega
$$

that is exactly what we did for the flat Minkowski metric. However, Lanczos has 
been studying the CC $\mathcal{D}_{2}$ of $\mathcal{D}_{1}$, ignoring that, contrary to the previous situation, $\mathcal{D}_{2}$ almost never comes from a linearization. It is therefore quite strange to discover that Lanczos never discovered that what he was doing with $\mathcal{D}_{1}$ and $\mathcal{D}_{2}$ while using quadratic Lagrangians in $R$, was exactly what is done in any textbook of elasticity or continum mechanics with $\mathcal{D}$ and $\mathcal{D}_{1}$ while using quadratic Lagrangians in $\Omega$ ([1] [7] [13] [16]). We do believe that Lanczos was too much obsessed by comparing $R$ in GR to $F$ in EM. Like in any good crime story, the solution will be given in the last section and could not have been given before by any classical approach.

- The last invited lecture published in 1962 by Lanczos on his potential theory is never quoted because it is in French. Comparing it with a commutative diagram in a recently published paper on gravitational waves ([18]), we suddenly understood the confusion made by Lanczos between Hodge duality and differential duality when he introduced his tentative 3-tensor potential. Our final purpose is thus to revisit the mathematical framework of Lanczos potential theory in the light of this comment,

\section{Mathematical Tools}

\subsection{Differential Sequences}

In view of the many examples that will be presented in this paper, it becomes clear that there is a need for classifying the properties of systems of PD equations in a way that does not depend on their presentations and this is the purpose of differential homological algebra along the scheme:

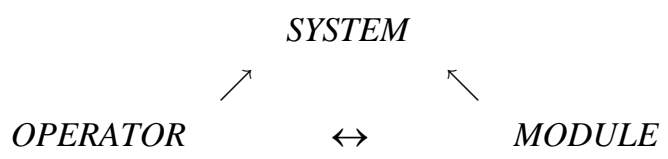

in order to show that certain concepts, which are clear in one framework, may become quite obscure in the others and conversely, like the formal integrability and torsion concepts for example.

When $E$ is a vector bundle over $X$ and we have a system of order $q$ on $E$, say $R_{q} \subset J_{q}(E)$, we can introduce the canonical projection

$\Phi: J_{q}(E) \rightarrow J_{q}(E) / R_{q}=F$ and define a linear differential operator $\mathcal{D}: E \rightarrow F: \xi(x) \rightarrow \eta^{\tau}(x)=a_{k}^{\tau \mu}(x) \partial_{\mu} \xi^{k}(x)$. When $\mathcal{D}$ is given, the compatibility conditions for solving $\mathcal{D} \xi=\eta$ can be described in operator form by $\mathcal{D}_{1} \eta=0$ and so on. In general, if a system is not formally integrable, it is possible to obtain a formally integrable system, having the same solutions, by "saturating" conveniently the given $\mathrm{PD}$ equations through the adjunction of new $\mathrm{PD}$ equations obtained by various prolongations/projections (PP) and such a procedure must absolutely be done before looking for the compatibility conditions ([21] [22]).

In order to study differential modules, we shall simply forget about changes of coordinates and only consider trivial bundles. If $K$ is a differential field with $n$ 
commuting derivations $\partial_{1}, \cdots, \partial_{n}$ (Say $\mathbb{Q}, \mathbb{Q}(a)$ or $\mathbb{Q}\left(x^{1}, \cdots, x^{n}\right)$ in the usual examples), we denote by $\operatorname{cst}(K)$ the subfield of constants of $K$, that is the set of elements killed by the $n$ derivations (Say $\mathbb{Q}$ in the usual examples). If $d_{1}, \cdots, d_{n}$ are formal derivatives (pure symbols in computer algebra packages!) which are only supposed to satisfy $d_{i} a=a d_{i}+\partial_{i} a$ in the operator sense for any $a \in K$, we may consider the (non-commutative) ring $D=K\left[d_{1}, \cdots, d_{n}\right]$ of differential operators with coefficients in $K$. If now $y=\left(y^{1}, \cdots, y^{m}\right)$ is a set of differential indeterminates, we let $D$ act formally on $y$ by setting $d_{\mu} y^{k}=y_{\mu}^{k}$ for any multi-index $\mu=\left(\mu_{1}, \cdots, \mu_{n}\right)$ and set $D y=D y^{1}+\cdots+D y^{m}$. We may also set $\Phi^{\tau} \equiv a_{k}^{\tau \mu} y_{\mu}^{k} \stackrel{d_{i}}{\rightarrow} d_{i} \Phi^{\tau} \equiv a_{k}^{\tau \mu} y_{\mu+1_{i}}^{k}+\partial_{i} a_{k}^{\tau \mu} y_{\mu}^{k}$ for $\tau=1, \cdots, p$. Denoting by $D \Phi$ the subdifferential module generated by all the given OD or PD equations and all their formal derivatives, we may finally introduce the $D$-module $M=D y / D \Phi$ by residue. Here we recall that $M$ is a module over a ring $A$ or an $A$-module if $\forall a \in A, \forall m, n \in M \Rightarrow a m, m+n \in M$. We may introduce as usual the torsion submodule $t(M)=\{m \in M \mid \exists 0 \neq a \in A, a m=0\}$ and we say that $M$ is a torsion module if $t(M)=M$ or that $M$ is torsion-free if $t(M)=0$.

It is not evident at all to exhibit the link existing between these two approaches and we proceed as follows. First of all, the ring $D$ is filtred by the order of the operators and we have the filtration or inductive limit

$0=D_{-1} \subset D_{0} \subset D_{1} \subset \cdots \subset D_{q} \subset \cdots \subset D_{\infty}=D$. Moreover, it is clear that $D$, as an algebra, is generated by $K=D_{0}$ and $T=D_{1} / D_{0}$ with $D_{1}=K \oplus T$ if we identify an element $\xi=\xi^{i} d_{i} \in T$ with the vector field $\xi=\xi^{i}(x) \partial_{i}$ of differential geometry, but with $\xi^{i} \in K$ now. As a byproduct, the differential module $D^{m}$ is also filtred by the order and we obtain an induced filtration or inductive limit $0=M_{-1} \subseteq M_{0} \subseteq M_{1} \subseteq \cdots \subseteq M_{q} \subseteq \cdots \subseteq M_{\infty}=M \quad$ with $d_{i} M_{q} \subseteq M_{q+1}$ provided by the prolongations. Now, if we suppose that the system $R_{q}=k e r(\Phi)$ is formally integrable (FI), that is all the OD or PD equations of order $q+r$ are obtained by using only $r$ prolongations, then we have the projective limit $R=R_{\infty} \rightarrow \cdots \rightarrow R_{q} \rightarrow R_{0} \rightarrow 0$ obtained by successive jet projections. We have the following crucial technical proposition ([13] [17]):

Proposition 2.A.1: $R=\operatorname{hom}_{K}(M, K)$ is a differential module for the Spencer operator and we have a bijective correspondence $M_{q} \leftrightarrow R_{q}=h_{K}\left(M_{q}, K\right)$ over $K$ because $K$ is a field.

Proof: for any $f \in R$ and $m \in M$, we may set for any $f \in R, m \in M$ :

$$
\begin{aligned}
& (a f)(m)=a(f(m))=f(a m), \forall a \in K, \\
& (\xi f)(m)=\xi(f(m))-f(\xi m), \forall \xi \in T
\end{aligned}
$$

and check that we have successively with $\xi \cdot a=\xi^{r} \partial_{r} a$ :

$$
\begin{aligned}
((\xi a) f)(m) & =(\xi(a f))(m) \\
& =\xi(a f(m))-a f(\xi m) \\
& =(\xi \cdot a) f(m)+a(\xi \cdot f(m))-f(a \xi m) \\
& =(\xi \cdot a) f(m)+((a \xi) f)(m)
\end{aligned}
$$


a result leading to $\xi a=a \xi+\xi \cdot a$ in the operator sense. Setting finally $f\left(y_{\mu}^{k}\right)=f_{\mu}^{k}$ with a slight abuse of notations when using the same notation $y_{\mu}^{k}$ for the residue instead of the standard $\bar{y}_{\mu}^{k}$. Setting $\mu+1_{i}=\left(\mu_{1}, \cdots, \mu_{i}+1, \cdots, \mu_{n}\right)$, it follows that $R$ is a differential module for the law:

$$
\left(d_{i} f\right)\left(y_{\mu}^{k}\right)=d_{i}\left(f\left(y_{\mu}^{k}\right)\right)-f\left(d_{i} y_{\mu}^{k}\right)=\partial_{i} f_{\mu}^{k}-f\left(y_{\mu+1_{i}}^{k}\right)=\partial_{i} f_{\mu}^{k}-f_{\mu+1_{i}}^{k}
$$

and we have $d_{i} d_{j} f=d_{j} d_{i} f=d_{i j} f, \forall f \in R$.

Q.E.D.

Through this paper, we shall only deal with linear differential operators. However, as explained in ([9] [23]), there is a nonlinear counterpart using the nonlinear Janet sequence coming from the Vessiot structure equations and a nonlinear Spencer sequence. The vertical machinery involved, that is a systematic use of fibered manifolds and vertical bundles, is much more difficult though we have chosen the notations of this paper in such a way that the interested reader may easily adapt them. As for the Vessiot structure equations first found in 1903 ([19]), they have been totally ignored during more than one century for reasons that are not scientific at all (See the original letters presented in [10] for explanations). Though we have written this paper in a rather self-contained way while using rather standard notations, the reader may refer to ([24] [25] [26]) for the differential geometric background, to ([27] [28]) for the elements of homological algebra needed through the various diagrames presented and to ([29] [30] [31]) for the main (difficult) concepts of differential homological algebra.

Collecting all the results so far obtained, if a differential operator $\mathcal{D}$ is given in the framework of differential geometry, we may keep the same operator matrix in the framework of differential modules which are left modules over the ring $D$ of linear differential operators. We may also apply duality over $D$, that is apply $\operatorname{hom}_{D}(\bullet, D)$, provided we deal now with right differential modules or use the operator matrix of $a d(\mathcal{D})$ and deal again with left differential modules obtained through the left $\leftrightarrow$ right conversion procedure. In actual practice, it is essential to notice that the new operator matrix may be quite different from the only transposed of the previous operator, even if we are dealing with constant coefficients.

Definition 2.A.2: If a differential operator $\xi \stackrel{\mathcal{D}}{\rightarrow} \eta$ is given, a direct problem is to find (generating) compatibility conditions (CC) as an operator $\eta \stackrel{\mathcal{D}_{1}}{\rightarrow} \zeta$ such that $\mathcal{D} \xi=\eta \Rightarrow \mathcal{D}_{1} \eta=0$. Conversely, given $\eta \stackrel{\mathcal{D}_{1}}{\rightarrow} \zeta$, the inverse problem will be to look for $\xi \stackrel{\mathcal{D}}{\rightarrow} \eta$ such that $\mathcal{D}_{1}$ generates the CC of $\mathcal{D}$ and we shall say that $\mathcal{D}_{1}$ is parametrized by $\mathcal{D}$... if such an operator $\mathcal{D}$ is existing!

Remark 2.A.3: Solving the direct problem (Janet, Spencer) is necessary for solving the inverse problem. However, though the direct problem always has a solution, the inverse problem may not have a solution at all and the case of the Einstein operator is one of the best non-trivial PD counterexamples (Compare 
[32] [33] [34]). It is rather striking to discover that, in the case of OD operators, it took almost 50 years to understand that the possibility to solve the inverse problem was equivalent to the controllability of the corresponding control system ([34]) and the situation is similar in GR as the above result has been first found in 1995 ([32]).

As $\operatorname{ad}(\operatorname{ad}(P))=P, \forall P \in D$, any operator is the adjoint of a certain operator and we recall that the double duality test needed in order to check whether $t(M)=0$ or not and to find out a parametrization if $t(M)=0$ when $M$ is defined by $\mathcal{D}_{1}$ has 5 steps which are drawn in the following diagram where $\operatorname{ad}(\mathcal{D})$ generates the $\mathrm{CC}$ of $\operatorname{ad}\left(\mathcal{D}_{1}\right)$ and $\mathcal{D}_{1}^{\prime}$ generates the $\mathrm{CC}$ of $\mathcal{D}=\operatorname{ad}(\operatorname{ad}(\mathcal{D}))$ :

$$
\begin{aligned}
& \zeta^{\prime} 5
\end{aligned}
$$

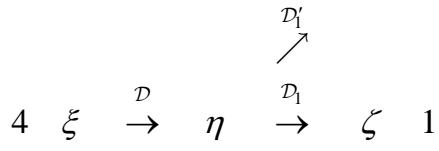

$$
\begin{aligned}
& 3 \quad v \stackrel{\operatorname{ad}(\mathcal{D})}{\leftarrow} \quad \mu \stackrel{\operatorname{ad}\left(\mathcal{D}_{1}\right)}{\leftarrow} \quad \lambda \quad 2
\end{aligned}
$$

Theorem 2.A.4: We have $\mathcal{D}_{1}$ parametrized by $\mathcal{D} \Leftrightarrow \mathcal{D}_{1} \simeq \mathcal{D}_{1}^{\prime} \Leftrightarrow t(M)=0 \Leftrightarrow \operatorname{ext}^{1}(N)=0$ in the differential module framework when $N$ is defined by $\operatorname{ad}\left(\mathcal{D}_{1}\right)$. These results do not depend on the finite free presentations of $M$ or $N$ (See [34] [35]) for more details).

Corollary 2.A.5: In the differential module framework, if $\stackrel{\mathcal{D}_{1}}{\rightarrow} F_{0} \stackrel{p}{\rightarrow} M \rightarrow 0$ is a finite free presentation of $M=\operatorname{coker}\left(\mathcal{D}_{1}\right)$ and we already know that $t(M)=0$ by using the preceding test and Theorem, then we may obtain an exact sequence $F_{1} \stackrel{\mathcal{D}_{1}}{\rightarrow} F_{0} \stackrel{\mathcal{D}}{\rightarrow} E$ of free differential modules where $\mathcal{D}$ is the parametrizing operator, both with an inclusion $M \subset E$ by chasing. However, there may exist other parametrizations $F_{1} \stackrel{\mathcal{D}_{1}}{\rightarrow} F_{0} \stackrel{\mathcal{D}^{\prime}}{\rightarrow} E^{\prime}$ called minimal parametrizations such that $\operatorname{coker}\left(\mathcal{D}^{\prime}\right)$ is a torsion module and we have thus $r k_{D}(M)=r k_{D}\left(E^{\prime}\right)$ (See [18] and [35]).

As shown by the next examples, the main difficulty met in OD or PD applications is that $\operatorname{ad}(\mathcal{D})$ may not be formally integrable at all, even if $\mathcal{D}$ is involutive (See [12] for other examples).

Example 2.A.6: (Double pendulum) If a rigid bar is able to move horizontally with reference position $x$ and we attach two pendula with respective length $l_{1}$ and $l_{2}$ making the (small) angles $\theta_{1}$ and $\theta_{2}$ with the vertical, the corresponding involutive control system is:

$$
d^{2} x+l_{1} d^{2} \theta^{1}+g \theta^{1}=0, \quad d^{2} x+l_{2} d^{2} \theta^{2}+g \theta^{2}=0
$$

where $g$ is the gravity. Multiplying these OD equations by two test functions $\lambda^{1}, \lambda^{2}$ and integrating by parts, we get the adjoint system:

$$
x \rightarrow d^{2} \lambda^{1}+d^{2} \lambda^{2}=0, \quad \theta^{1} \rightarrow l_{1} d^{2} \lambda^{1}+g \lambda^{1}=0, \quad \theta^{2} \rightarrow l_{2} d^{2} \lambda^{2}+g \lambda^{2}=0
$$

Multiplying the second equation by $l_{2}$, the third by $l_{1}$ while using the first, we 
obtain the zero order OD equation $l_{2} \lambda^{1}+l_{1} \lambda^{2}=0$. Differentiating twice this time and substituting, we obtain the new zero order OD equation $\left(l_{2} / l_{1}\right) \lambda^{1}+\left(l_{1} / l_{2}\right) \lambda^{2}=0$. The determinant of the system of two zero order equations is then seen to be exactly $l_{1}-l_{2}$. It follows that the system is controllable if and only if $l_{1}$ is different from $l_{2}$, a fact that the reader can check easily when moving the bar conveniently. If one length depends on time, the corresponding controllability condition cannot be obtained without computer algebra, even on such an elementary control system. The totally unexpected fourth order parametrization of the control system when it is controllable is:

- $l_{1} \neq l_{2}$ :

$$
\left\{\begin{array}{l}
-l_{1} l_{2} d^{4} \phi-g\left(l_{1}+l_{2}\right) d^{2} \phi-g^{2} \phi=x \\
l_{2} d^{4} \phi+g d^{2} \phi=\theta_{1} \\
l_{1} d^{4} \phi+g d^{2} \phi=\theta_{2}
\end{array}\right.
$$

- $l_{1}=l_{2}=l, \theta=\theta_{1}-\theta_{2} \Rightarrow l d^{2} \theta+g \theta=0, \theta(0)=0, d \theta(0)=0 \Rightarrow \theta(t)=0$.

It follows that the controllability of a control system is a "built in" property of this system that does not depend on the choice of the control variables, contrary to a tradition still existing in the control community (See Zbl 1079.93001 for a review). We invite the reader to use the Kalman approach that can be found in any control textbook today and to compare (See [12] or [34] for details).

Example 2.A.7: (Einstein equations) If $\mathcal{D}_{1}$ is the Einstein operator which is self-adjoint, then $\operatorname{ad}\left(\mathcal{D}_{1}\right)$ is also the Einstein operator, $\operatorname{ad}(\mathcal{D})$ is the Cauchy operator and $\mathcal{D}$ is thus the Killing operator. It follows that $\mathcal{D}_{1}^{\prime}$ is the Riemann operator according to the Introduction. Using the previous theorem, any component of the Weyl tensor becomes a torsion element killed by the Dalembert operator as a "modern" description of the so-called Lichnerowicz waves (as they are called in France!) ([16]).

\subsection{Variational Calculus}

Having in mind "Optimal Control Theory" while using the notations of the previous Formal Test, let us assume that the two differential sequences:

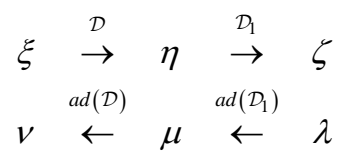

are formally exact, that is $\mathcal{D}_{1}$ generates the CC of $\mathcal{D}$ and $\operatorname{ad}(\mathcal{D})$ generates the CC of $\operatorname{ad}\left(\mathcal{D}_{1}\right)$, namely $\xi$ is a potential for $\mathcal{D}_{1}$ and $\lambda$ is a potential for $\operatorname{ad}(\mathcal{D})$. We may consider a variational problem for a cost function or lagrangian $\varphi(\eta)$ under the linear OD or PD constraint described by $\mathcal{D}_{1} \eta=0$.

- Introducing convenient Lagrange multipliers $\lambda$ while setting $\mathrm{d} x=\mathrm{d} x^{1} \wedge \cdots \wedge \mathrm{d} x^{n}$ for simplicity, we must vary the integral:

$$
\Phi=\int\left[\varphi(\eta)-\lambda \mathcal{D}_{1} \eta\right] \mathrm{d} x \Rightarrow \delta \Phi=\int\left[(\partial \varphi(\eta) / \partial \eta) \delta \eta-\lambda \mathcal{D}_{1} \delta \eta\right] \mathrm{d} x
$$

Integrating by parts, we obtain the Euler-Lagrange (EL) equations: 


$$
\partial \varphi(\eta) / \partial \eta=\operatorname{ad}\left(\mathcal{D}_{1}\right) \lambda
$$

to which we have to add the constraint $\mathcal{D}_{1} \eta=0$ obtained by varying $\lambda$ independently. If $\operatorname{ad}\left(\mathcal{D}_{1}\right)$ is an injective operator, in particular if $\mathcal{D}_{1}$ is formally surjective (no CC) while $n=1$ as inOD optimal control and $M$ is torsion-free, thus free ([12]) or $n \geq 1$ and $M$ is projective, then one can obtain $\lambda$ explicitly and eliminate it by substitution. Otherwise, using the CC $\operatorname{ad}(\mathcal{D})$ of $\operatorname{ad}\left(\mathcal{D}_{1}\right)$, we have to study the formal integrability of the combined system:

$$
\operatorname{ad}(\mathcal{D}) \partial \varphi(\eta) / \partial \eta=0, \quad \mathcal{D}_{1} \eta=0
$$

which may be a difficult task ([12], Introduction and Chapter VI).

- However, we may also transform the given variational problem with constraint into a variational problem without any constraint if and only if the differential constraint can be parametrized. Using the parametrization of $\mathcal{D}_{1}$ by $\mathcal{D}$, we may vary the integral:

$$
\Phi=\int \varphi(\mathcal{D} \xi) \mathrm{d} x \Rightarrow \delta \Phi=\int(\partial \varphi(\eta) / \partial \eta) \mathcal{D} \delta \xi \mathrm{d} x
$$

whenever $\eta=\mathcal{D} \xi$ and integrate by parts for arbitrary $\delta \xi$ in order to obtain the EL equations:

$$
\operatorname{ad}(\mathcal{D}) \partial \varphi(\eta) / \partial \eta=0, \quad \eta=\mathcal{D} \xi
$$

in a coherent way with the previous approach.

As a byproduct, if the field equations $\mathcal{D}_{1} \eta=0$ can be parametrized by a potential $\xi$ through the formula $\mathcal{D} \xi=\eta$, then the induction equations $\operatorname{ad}(\mathcal{D}) \mu=v$ can be obtained by duality in a coherent way with the double duality test, on the condition to know what sequence must be used. However, we have yet proved in ([9] [10] [13] [36]) that the Cauchy stress equations must be replaced by the Cosserat couple-stress equations and that the Janet sequence (only used in this paper) must be thus replaced by the Spencer sequence. Accordingly, the work of Lanczos ([2] [3] [4] [5]) and followers ([37] [38] [39] [40] [41]), using either exterior calculus, Janet and Gröbner bases or Pommaret bases, has been based on a confusion between fields and inductions on one side, but also between the Janet sequence and the Spencer sequence. By chance, as we always refer to intrinsic concepts like the extension modules that do not depend on the differential sequence used, all the results that will be presented can be adapted at once to the systematic use of the Spencer sequence in place of the Janet sequence.

\section{Riemann/Lanczos Problem}

The last invited lecture published in 1962 by Lanczos on his potential theory is never quoted because it is in French ([5]). Comparing it with a commutative diagram in a recently published paper on gravitational waves ([16]), we suddenly understood the confusion made by Lanczos between Hodge duality and differential duality. Our purpose is thus to revisit the mathematical framework of Lanczos potential theory in the light of this comment, getting closer to the for- 
mal theory of Lie pseudogroups through double differential duality and the construction of finite length differential sequences for Lie operators.

When $K$ is a differential field containing the field $\mathbb{Q}$ of rational numbers and $\left(d_{1}, \cdots, d_{n}\right)$ are commuting formal derivatives, we may introduce the ring (which is even an integral domain) $D=K\left[d_{1}, \cdots, d_{n}\right]=K[d]$ of differential operators with coefficients in $K$. Accordingly, if a differential module $M$ with torsion submodule $t(M)$ is defined by an operator $\mathcal{D}$ with coefficients in $K$, we may introduce the differential extension modules ext ${ }^{0}(M)=\operatorname{hom}_{D}(M, D)$ and ext $^{i}(M)=$ ext $_{D}^{i}(M, D)$ for $i=1, \cdots, n$. We have the long exact ker/coker long exact sequence of (left) differential modules (See [12] or [34] for details):

$$
0 \rightarrow \operatorname{ext}^{1}(N) \rightarrow M \stackrel{\varepsilon}{\rightarrow} \operatorname{hom}_{D}\left(\operatorname{hom}_{D}(M, D), D\right) \rightarrow \operatorname{ext}^{2}(N) \rightarrow 0
$$

where the morphism $\varepsilon$ is defined by $(\varepsilon(m))(f)=f(m), \forall m \in M, \forall f \in \operatorname{hom}_{D}(M, D)$ and the adjoint differential module $N=\operatorname{ad}(M)$ is defined by the adjoint operator $\operatorname{ad}(\mathcal{D})$. Then $M$ is torsion-free if and only if $t(M)=e^{1} t^{1}(N)=0$, that is $\varepsilon$ is a monomorphism or, equivalently, $\mathcal{D}$ can be parametrized by the operator $\mathcal{D}_{-1}$ when $\operatorname{ad}\left(\mathcal{D}_{-1}\right)$ generates the compatibility conditions (CC) of $\operatorname{ad}(\mathcal{D})$. Finally $M$ is reflexive if and only if, in addition, $\varepsilon$ is an epimorphism, that is we have also ext ${ }^{2}(N)=0$ or, equivalently, $\mathcal{D}_{-1}$ can be parametrized again by $\mathcal{D}_{-2}$ when $\operatorname{ad}\left(\mathcal{D}_{-2}\right)$ generates the CC of $\operatorname{ad}\left(\mathcal{D}_{-1}\right)$. As we have $\operatorname{ad}(\operatorname{ad}(\mathcal{D}))=\mathcal{D}$ and though this is not evident at first sight by exchanging $M$ with $N$, we may also say that $\operatorname{ext}^{1}(M)=0$ if $\operatorname{ad}(\mathcal{D})$ generates the CC of $\operatorname{ad}\left(\mathcal{D}_{1}\right)$ whenever $\mathcal{D}_{1}$ generates the CC of $\mathcal{D}$ and, similarly, that $\operatorname{ext}^{2}(M)=0$ when $\operatorname{ad}\left(\mathcal{D}_{1}\right)$ generates the CC of $\operatorname{ad}\left(\mathcal{D}_{2}\right)$ whenever $\mathcal{D}_{2}$ generates the CC of $\mathcal{D}_{1}$. We shall provide an explicit description of the potentials allowing to parametrize the Riemann and the Weyl operators in arbitrary dimension, both with their respective adjoint operators.

We now consider with details the Riemann/Lanczos problem which is at the same time the simplest of the two Lanczos problems as it can be solved in arbitrary dimension $n \geq 2$ but is also an example of the successive confusing works that have been done during the last fifty years as we already said. According to the last Section, the starting motivation seems absolutely natural at first. Indeed, considering the Killing operator $\mathcal{D}: \xi \rightarrow \mathcal{L}(\xi) \omega=\Omega \in S_{2} T^{*}=F_{0}$ where $\mathcal{L}(\xi)$ is the Lie derivative with respect to $\xi$ and $\omega \in S_{2} T^{*}$ is a nondegenerate metric with $\operatorname{det}(\omega) \neq 0$. Accordingly, it is a Lie operator with $\mathcal{D} \xi=0, \mathcal{D} \eta=0 \Rightarrow \mathcal{D}[\xi, \eta]=0$ and we denote simply by $\Theta \subset T$ the set of solutions with $[\Theta, \Theta] \subset \Theta$. Now, as we have explained many times, the main problem is to describe the CC of $\mathcal{D} \xi=\Omega \in F_{0}$ in the form $\mathcal{D}_{1} \Omega=0$ by introducing the so-called Riemann operator $\mathcal{D}_{1}: F_{0} \rightarrow F_{1}$, using the standard notations that can be found at length in our many books ([8]-[13]) or papers ([14] [42]). We advise the reader to follow closely the next lines and to imagine why it will not be possible to repeat them for studying the Weyl/Lanczos problem. Introducing the Levi-Civita isomorphism $j_{1}(\omega)=\left(\omega, \partial_{x} \omega\right) \simeq(\omega, \gamma)$ and the 
Christoffel symbols $\gamma_{i j}^{k}=\frac{1}{2} \omega^{k r}\left(\partial_{i} \omega_{r j}+\partial_{j} \omega_{i r}-\partial_{r} \omega_{i j}\right)$ where $\left(\omega^{r s}\right)$ is the inverse matrix of $\left(\omega_{i j}\right)$, we get $R_{2} \subset J_{2}(T)$ :

$$
\left\{\begin{array}{l}
\Omega_{i j} \equiv \omega_{r j}(x) \xi_{i}^{r}+\omega_{i r}(x) \xi_{j}^{r}+\xi^{r} \partial_{r} \omega_{i j}(x)=0 \\
\Gamma_{i j}^{k} \equiv \xi_{i j}^{k}+\gamma_{r j}^{k}(x) \xi_{i}^{r}+\gamma_{i r}^{k}(x) \xi_{j}^{r}+\gamma_{i r}^{k}(x) \xi_{j}^{r}-\gamma_{i j}^{r}(x) \xi_{r}^{k}+\xi^{r} \partial_{r} \gamma_{i j}^{k}(x)=0
\end{array}\right.
$$

if we use jet coordinates with sections $\xi_{q}: x \rightarrow\left(\xi^{k}(x), \xi_{i}^{k}(x), \xi_{i j}^{k}(x), \cdots\right)$ transforming like $j_{q}(\xi): x \rightarrow\left(\xi^{k}(x), \partial_{i} \xi^{k}(x), \partial_{i j} \xi^{k}(x), \cdots\right)$. The system $R_{1} \subset J_{1}(T)$ has a symbol $g_{1} \simeq \wedge^{2} T^{*} \subset T^{*} \otimes T$ depending only on $\omega$ with $\operatorname{dim}\left(g_{1}\right)=n(n-1) / 2$ and is finite type because its first prolongation is $g_{2}=0$. It cannot be thus involutive as can be seen directly on the following Janet board for finding a Pommaret basis when $n=2$ and $\omega$ is the euclidean metric:

$$
\left\{\begin{array}{l|l|}
\xi_{2}^{2}=0 \\
\xi_{2}^{1}+\xi_{1}^{2}=0 \\
\xi_{1}^{1}=0
\end{array}\right.
$$

Indeed, the only dot appearing in the board cannot provide any CC for the symbol $g_{1}$ and we have therefore the short exact sequence:

$$
0 \rightarrow g_{2} \rightarrow S_{2} T^{*} \otimes T \rightarrow T^{*} \otimes F_{0} \rightarrow 0
$$

by using the fact that $g_{2}=0$ and counting the common dimension $n^{2}(n+1) / 2$, because an epimorphism between two spaces of the same dimension is also a monomorphism and thus an isomorphism. Accordingly, we need to use one additional prolongation and arrive to the:

- First comment. Using now one of the main results to be found in $([8], \ldots$ [12]), we know that, when $R_{1}$ is formally integrable, then the CC of $\mathcal{D}$ are of order $s+1$ where $s$ is the number of prolongations needed in order to get an involutive symbol, that is $s=1$ in the present situation, a result that should lead to CC of order 2 if $R_{1}$ were formally integrable.

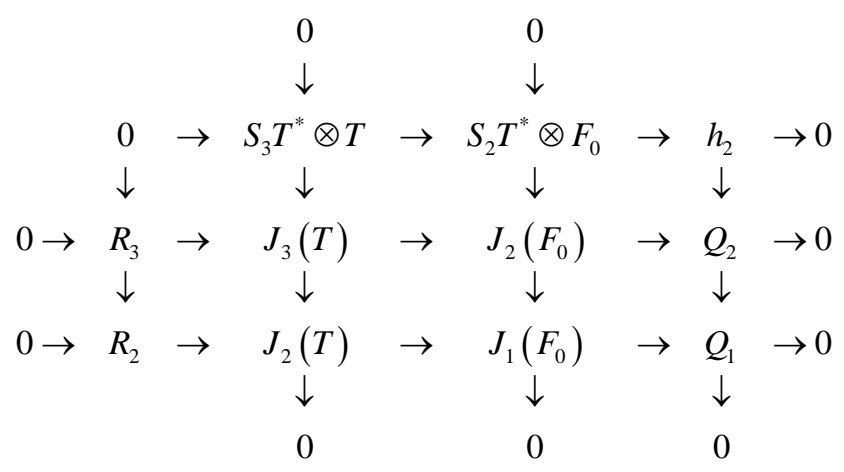

As $Q_{1}=0$ by counting the dimensions with $\operatorname{dim}\left(R_{2}\right)=n+n(n-1) / 2=n(n+1) / 2$ and $g_{3}=0$, we get $\operatorname{dim}\left(Q_{2}\right) \leq \operatorname{dim}\left(h_{2}\right)=n^{2}(n+1)^{2} / 4-n^{2}(n+1)(n+2) / 6=n^{2}\left(n^{2}-1\right) / 12$. Hence, we understand that the number of CC $\mathcal{D}_{1}$ of $\mathcal{D}$ is equal to the number of components of the Riemann tensor if and only if $R_{2}$ is formally integrable, that 
is if and only if $\omega$ has constant Riemannian curvature, a result first found by L.P. Eisenhart in 1926 ([20]) though in a different setting (See [8] for an explicit modern proof). Such a necessary condition for constructing an exact differential sequence could not have been used by Lanczos because the work of Spencer has only been known after 1970 ([24] [26]). Otherwise, if the metric does not satisfy this condition, CC may exist by using the Petrov classification but have no link with the Riemann tensor ([22]). We may therefore define the model vector bundle $F_{1}$ with $\operatorname{dim}\left(F_{1}\right)=n^{2}\left(n^{2}-1\right) / 12$ in the sense of Lanczos by the short exact sequence:

$$
0 \rightarrow S_{3} T^{*} \otimes T \rightarrow S_{2} T^{*} \otimes F_{0} \rightarrow F_{1} \rightarrow 0
$$

A result leading to the operator $D_{1}: F_{0} \stackrel{j_{2}}{\rightarrow} J_{2}\left(F_{0}\right) \rightarrow F_{1}$ and the:

- Second comment. Applying the Spencer operator $\delta$ to the top line of the preceding diagram, we get the commutative diagram:

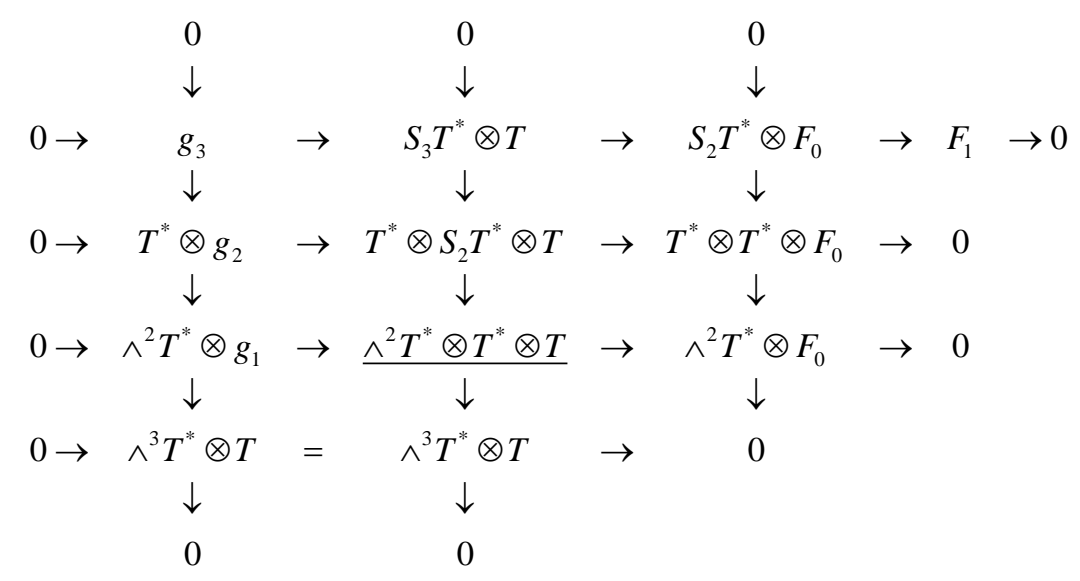

Using a diagonal chase, we discover that $F_{1}$ is just the Spencer $\delta$-cohomology $H^{2}\left(g_{1}\right)$ at $\wedge^{2} T^{*} \otimes g_{1}$ along the following short exact sequence:

$$
0 \rightarrow F_{1} \rightarrow \wedge^{2} T^{*} \otimes g_{1} \stackrel{\delta}{\rightarrow} \wedge^{3} T^{*} \otimes T \rightarrow 0
$$

because $g_{2}=0$ and we get the striking formula where the + signs have been replaced by signs:

$$
\operatorname{dim}\left(F_{1}\right)=n^{2}(n-1)^{2} / 4-n^{2}(n-1)(n-2) / 6=n^{2}\left(n^{2}-1\right) / 12
$$

This result, first found by the author in 1978 ([8]), clearly exhibit the two well known algebraic properties of the Riemann tensor. We now understand that Lanczos had in mind to linearize the Riemann tensor over the Minkowski metric, exactly like in $G R$, in order to construct a Lagrangian as a function of the corresponding linearization $R_{l, i j}^{k}$ of the Riemann tensor $\rho_{l, i j}^{k}$, transforming the usual variational problem into a variational with a differential constraint described by the Bianchi identities leading to the operator $\mathcal{D}_{2}$. As an equivalent alternative approach, his idea was to consider the curvature as a field by itself and construct the lagrangian on this field like in EM while adding the Bianchi identities as a differential constraint by using as many Lagrange multiplier as the 
number of Bianchi identities, a number not known by combinatorics at the time Lanczos was writing, a result leading to the:

- Third comment. Lanczos, who also knew continuum mechanics as an engineer, just copied the way used in elasticity (EL) and in electromagnetism (EM), for example introducing a Lagrangian as a function of the deformation $\varepsilon=(1 / 2) \Omega$ while adding a differential constraint described by the vanishing linearized Rieman tensor with therefore as many Lagrange multipliers as the number $n^{2}\left(n^{2}-1\right) / 12$ of components of the Riemann tensor. It is crucial to notice that the same differential sequence is used one step before, that is with $\mathcal{D}$ and $\mathcal{D}_{1}$ while he was dealing with $\mathcal{D}_{1}$ and $\mathcal{D}_{2}$ previously, that is one step ahead in the sequence. We have proved recently that such a procedure is in total contradiction with the piezoelectricity and photoelasticity existing between EL and EM (See the picture in [7]). It thus remains to exhibit the Bianchi operator exactly as we did for the Riemann operator, with the same historical comments already provided. However, now we know that $R_{1}$ is formally integrable (otherwise nothing could be achieved and we should start with a smaller system!), the construction of the linearized Janet-type differential sequence as a strictly exact differential sequence but not an involutive differential sequence because the system $R_{1}$ and thus the first order operator $\mathcal{D}$ are formally integrable though not involutive as $g_{1}$ is finite type with $g_{2}=0$ but not involutive. Doing one more prolongation only, we obtain the first order Bianchi CC from $F_{2}$ in the following long exact symbol sequence (See the details below):

$$
0 \rightarrow S_{4} T^{*} \otimes T \rightarrow S_{3} T^{*} \otimes F_{0} \rightarrow T^{*} \otimes F_{1} \rightarrow F_{2} \rightarrow 0
$$

or from the short exact sequence:

$$
0 \rightarrow F_{2} \rightarrow \wedge^{3} T^{*} \otimes g_{1} \stackrel{\delta}{\rightarrow} \wedge^{4} T^{*} \otimes T \rightarrow 0
$$

showing that $F_{2}=H^{3}\left(g_{1}\right)$ ([8] [9] [13]). We have in particular for $n \geq 3$ :

$$
\begin{aligned}
& \operatorname{dim}\left(F_{2}\right) \\
& =n^{2}(n-1)^{2}(n-2) / 12-n^{2}(n-1)(n-2)(n-3) / 24 \\
& =n^{2}(n+1)(n+2)(n+3) / 24+n^{3}\left(n^{2}-1\right) / 12-n^{2}(n+1)(n+2)(n+3) / 24 \\
& =n^{2}\left(n^{2}-1\right)(n-2) / 24
\end{aligned}
$$

and thus $\operatorname{dim}\left(F_{2}\right)=(4 \times 6)-(1 \times 4)=(16 \times 15 \times 2) / 24=20$ when $n=4$, a result leading to:

- Fourth comment. (Double Hodge duality) For an arbitrary $n$, it is not possible to recognize that one of the algebraic conditions for the Bianchi identity comes from the Spencer $\delta$-map and is again an epimorphism as it was before for defining $F_{1}$, a result obtained by chasing in the commutative diagram obtained by applying $\delta$ to the long exact symbol sequence finishing with $F_{2}$. It is not evident at all to discover that the modern description of the model vector bundle $F_{2}$ is just equivalent to the one provided by Lanczos 
but only for $n=4$. For this, using local coordinates, we have the 4 linear equations with $i=1,2,3,4$ :

$$
B_{1,234}^{i}-B_{2,341}^{i}+B_{3,412}^{i}-B_{4,123}^{i}=0
$$

to be compared with the 4 equations for the Lanczos tensor with $L_{i j, k}+L_{j i, k}=0$, namely:

$$
L_{i j, k}+L_{j k, i}+L_{k i, j}=0
$$

Before reading the next lemma, we invite the reader to prove ... that they are identica!

Lemma 3.1: These two equations are identical onlywhen $n=4$.

Proof. Using Hodge duality a first time, we may rewrite the first ones in the form:

$$
B_{1,1}^{i}+B_{2,2}^{i}+B_{3,3}^{i}+B_{4,4}^{i}=0, \quad \forall i=1,2,3,4
$$

Lowering the index $i$ by means of the Eucldean metric for simplicity and setting $i=4$, we get:

$$
B_{44,4}=0 \Rightarrow B_{41,1}+B_{42,2}+B_{43,3}=0
$$

Using again the Hodge duality but setting now $B_{41,1}=L_{23,1}$ and so on, we get:

$$
L_{23,1}+L_{31,2}+L_{12,3}=0
$$

that is exactly the Lanczos formula, a result showing that, for $n=4$ only, we discover that $L \simeq B \in \wedge^{3} T^{*} \otimes g_{1}$ are both killed by $\delta$.

We are thus able to exhibit the Lanczos potential $L \in \wedge^{2} T^{*} \otimes T^{*}$ as a 3-tensor satisfying:

$$
L_{i j, k}+L_{j i, k}=0, L_{i j, k}+L_{j k, i}+L_{k i, j}=0
$$

in the short exact sequence $0 \rightarrow \delta\left(T^{*} \otimes S_{2} T^{*}\right) \rightarrow \wedge^{2} T^{*} \otimes T^{*} \stackrel{\delta}{\rightarrow} \wedge^{3} T^{*} \rightarrow 0$ but this result does not provide any potential because ... the adjoint sequence is going backwards!.

Q.E.D.

Using adjoint operators and adjoint bundles while setting $\operatorname{ad}(E)=\wedge^{n} T^{*} \otimes E^{*}$ when $E$ is a vector bundle over $X$ and using the Hodge duality, we obtain the short exact sequences with arrows reversed:

$$
0 \leftarrow \operatorname{ad}\left(F_{2}\right) \leftarrow \wedge^{n-3} T^{*} \otimes g_{1}^{*} \leftarrow \delta^{*} \wedge^{n-4} T^{*} \otimes T^{*} \leftarrow 0
$$

as a way to describe the Lagrange multiplier $\lambda \in \operatorname{ad}\left(F_{2}\right)$ in arbitrary dimension.

These results are leading to the:

- Fifth comment: The div-type operator induced (on the right) by the Bianchi operator has strictly nothing to do with the Cauchy operator (namely ad(Killing) on the left), contrary to what is still believed in GR. In addition, we have the:

- Sixth comment. We have proved in ([38] [39] [40]) that the usual Cauchy 
stress equations must be replaced by the Cosserat couple-stress equations or, equivalently, that the Janet sequence must be replaced by the Spencer sequence in a coherent way with the couplings existing between EL and EM. It is also important to notice that, in the non-linear framework, there is no analogue of $\mathcal{D}_{2}$ in the nonlinear Janet sequence or of $D_{3}$ in the nonlinear Spencer sequence, a redhibitory reason leading to use only $\mathcal{D}$ and $\mathcal{D}_{1}$ or $D_{1}$ and $D_{2}$ both with their formal adjoints.

As a way to conclude this example, we may say that, for any $n \geq 3$, the Riemann operator $\mathcal{D}_{1}$ is parametrizing the Bianchi operator $\mathcal{D}_{2}$ while the operator $\operatorname{ad}\left(\mathcal{D}_{2}\right)$ is parametrizing the operator $\operatorname{ad}\left(\mathcal{D}_{1}\right)$. Nevertheless, according to ([15]), there may exist minimal parametrizations of $\mathcal{D}_{2}$ with a lower number of potentials equal to $\operatorname{dim}(T)+\operatorname{dim}\left(F_{1}\right)-\operatorname{dim}\left(F_{0}\right)$, thus $4+20-10=14=20-6$ when $n=4$ because of the Euler-Poincaré characteristic $4-10+20-20+6=0$ (See [18]).

Remark 3.2: Lanczos has been trying in vain to do for the Bianchi operator what he did for the Riemann operator, a useless but possible "shift by one step to the right" and to do for the Weyloperator what he did for the Riemann operator. However, we shall discover that the dimension $n=4$, which is particularly "fine" for the classical Killing sequence, is particularly "bad" for the conformal Killing sequence, a result not known after one century because it cannot be understood without using the Spencer $\delta$-cohomology in the following commutative diagram which is explaining therefore what we shall call the "Lanczos secret". Following ([21]) and the fact that the two central vertical $\delta$-sequences are exact, this diagram allows to construct the Bianchi operator $\mathcal{D}_{2}: F_{1} \rightarrow F_{2}$ as generating CC for the Riemann operator $\mathcal{D}_{1}: F_{0}=S_{2} T^{*} \rightarrow F_{1}$ defined by a similar diagram and thus only depends on the symbol $g_{1}$. For the reader not familiar with homological algebra, we provide below the main diagram allowing to construct the $B i$ anchi operator both with the corresponding fiber dimensions when $n=4$. In this commutative diagram, all the rows are exact and the columns are exact but eventually the left one:

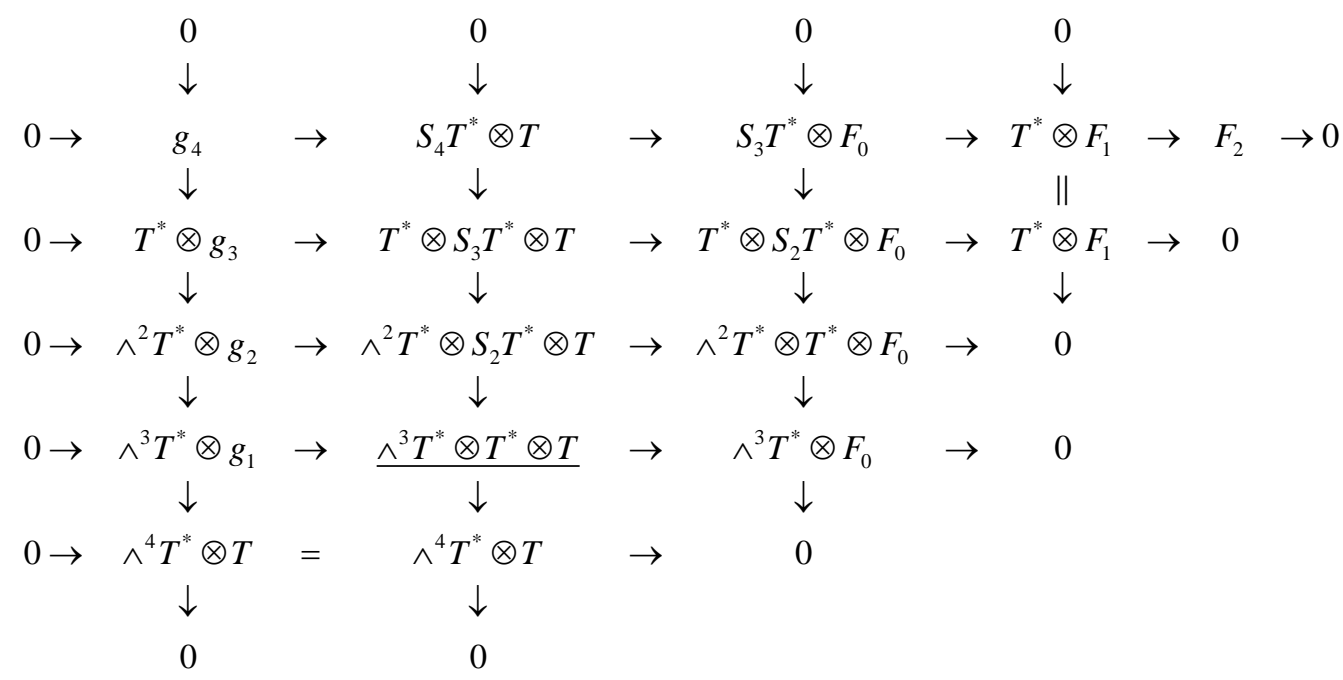




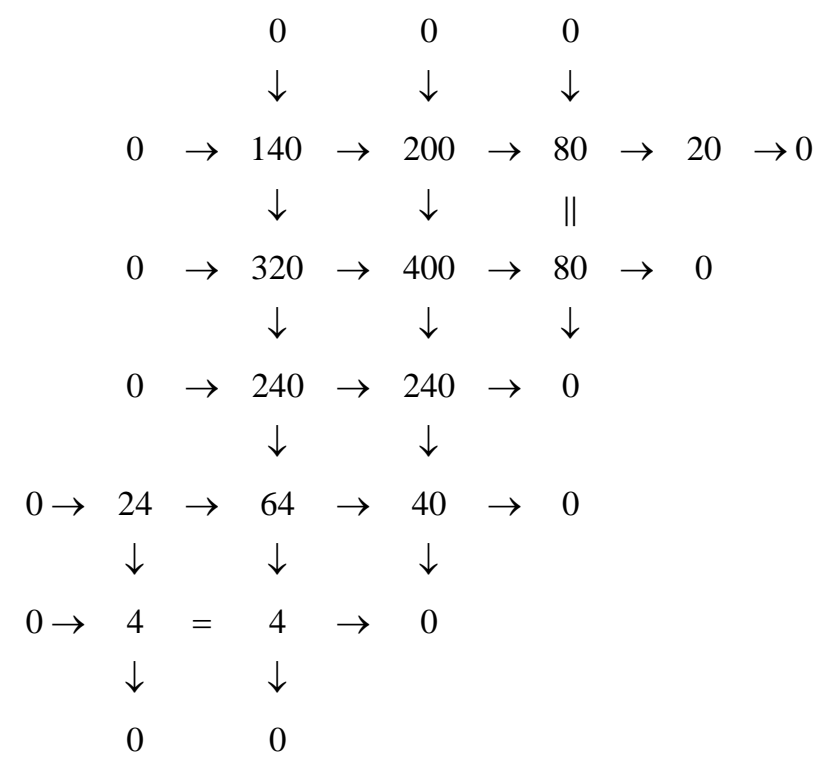

Using the Spencer cohomology at $\wedge^{2} T^{*} \otimes g_{1}$, the vector bundle $F_{1}=H^{2}\left(g_{1}\right)$ in this diagram or Riemann candidate in the language of Lanczos, is defined by the short exact sequence:

$$
\begin{aligned}
& 0 \rightarrow \quad F_{1} \rightarrow \wedge^{2} T^{*} \otimes g_{1} \stackrel{\delta}{\rightarrow} \wedge^{3} T^{*} \otimes T \quad \rightarrow 0 \\
& 0 \rightarrow 20 \rightarrow \quad 36 \quad \stackrel{\delta}{\rightarrow} \quad 16 \quad \rightarrow 0
\end{aligned}
$$

All the vertical down arrows are $\delta$-maps of Spencer and all the vertical columns are exact but the first, which may not be exact only at $\wedge^{3} T^{*} \otimes g_{1}$ with cohomology equal to $H^{3}\left(g_{1}\right)$ because we have:

$$
\begin{aligned}
& g_{1}=\left\{\xi_{i}^{k} \in T^{*} \otimes T \mid \omega_{r j} \xi_{i}^{r}+\omega_{i r} \xi_{j}^{r}=0\right\} \simeq \wedge^{2} T^{*} \subset T^{*} \otimes T \\
& \stackrel{\operatorname{det}(\omega) \neq 0}{\Rightarrow} g_{2}=0 \Rightarrow g_{3}=0 \Rightarrow g_{4}=0
\end{aligned}
$$

A snake-type chase similarly provides the identification $F_{2}=H^{3}\left(g_{1}\right)$ while using again the Spencer cohomology at $\wedge^{3} T^{*} \otimes g_{1}$. The vector bundle $F_{2}$ providing the Bianchi identities is thus defined by the exactness of the top row of the preceding diagram or, equivalently, using the left column, by the short exact sequence:

$$
\begin{aligned}
& 0 \rightarrow \quad F_{2} \rightarrow \wedge^{3} T^{*} \otimes g_{1} \stackrel{\delta}{\rightarrow} \wedge^{4} T^{*} \otimes T \quad \rightarrow 0 \\
& 0 \rightarrow 20 \rightarrow \quad 24 \quad \stackrel{\delta}{\rightarrow} \quad 4 \quad \rightarrow 0
\end{aligned}
$$

We conclude this Remark by saying that it is not even easy to discover that the bottom $\delta$-map in the first column on the left is an epimorphism. In order to convince the reader of the powerfulness of these new methods, this result is left a an exercise (Hint: prove that:

$$
\wedge^{3} T^{*} \otimes T^{*} \otimes T \simeq \wedge^{3} T^{*} \otimes g_{1}+\delta\left(\wedge^{2} T^{*} \otimes S_{2} T^{*} \otimes T\right)
$$


by using a circular counterclockwise chase and that $\left.F_{2} \simeq \wedge^{3} T^{*} \otimes g_{1} \cap \delta\left(\wedge^{2} T^{*} \otimes S_{2} T^{*} \otimes T\right)\right)$.

Starting with the (classical) Killing operator $K: T \rightarrow S_{2} T^{*}$ defined by $\xi \rightarrow L(\xi) \omega$, we obtain successively the following differential sequences for various dimensions:

$$
\begin{aligned}
& n=2 \quad 2 \underset{1}{\stackrel{K}{\rightarrow}} 3 \underset{2}{\stackrel{R}{\rightarrow}} 1 \rightarrow 0 \\
& n=3 \quad 3 \underset{1}{\stackrel{K}{\rightarrow}} \quad 6 \underset{2}{\stackrel{R}{\rightarrow}} \quad 6 \quad \underset{1}{\stackrel{B}{\rightarrow}} \quad 3 \quad \rightarrow \quad 0 \\
& n=4 \quad 4 \underset{1}{\stackrel{K}{\rightarrow}} \quad 10 \underset{2}{\stackrel{R}{\rightarrow}} \quad 20 \underset{1}{\stackrel{B}{\rightarrow}} \quad 20 \underset{1}{\rightarrow} \quad 6 \quad \rightarrow \quad 0
\end{aligned}
$$

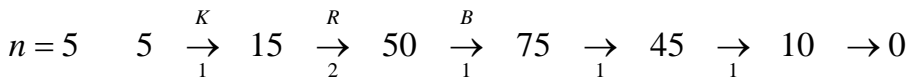

For example, we have the Euler-Poincaré characteristic:

$r k_{D}(M)=4-10+20-20+6=0$ when $n=4$ or $5-15+50-75+45-10=0$ when $n=5$.

Setting successively $\mathcal{D}=K, \mathcal{D}_{1}=R, \mathcal{D}_{2}=B$ and so on, it follows therefore from the previous study that each operator is parametrizing the following one. Applying double duality and introducing the respective adjoint operators, then $\operatorname{ad}\left(\mathcal{D}_{2}\right)$ is parametrizing the Beltrami operator ad $(\operatorname{Riemann})=\operatorname{ad}\left(\mathcal{D}_{1}\right)$ with (canonical) potentials called Lanczos only when $n=4$ while $\operatorname{ad}\left(\mathcal{D}_{1}\right)$ is parametrizing the Cauchy operator $\operatorname{ad}(\mathcal{D})$ with (canonical) potentials called Airy, Beltrami, ... ([15] [18]). It must be finally noticed that ad(Ricci) is also parametrizing the Cauchyoperator ([16] [18]).

\section{Weyl/Lanczos Problem}

Starting now afresh with the conformal Killing operator $C K$ such that $\mathcal{L}(\xi) \omega=A(x) \omega$ or, equivalently, introducing the metric density $\hat{\omega}_{i j}=\omega_{i j}|\operatorname{det}(\omega)|^{-\frac{1}{n}}$, we have a new operator $C K: T \rightarrow\left\{\Omega \in S_{2} T^{*} \mid \operatorname{tr}(\Omega)=\omega^{i j} \Omega_{i j}=0\right\} \quad$ defined by $\xi \rightarrow \mathcal{L}(\xi) \hat{\omega}$ and we obtain successively the following differential sequences for various dimensions $n \geq 3$ ([43]):

$$
\begin{aligned}
& n=3 \quad 3 \underset{1}{\stackrel{C K}{\rightarrow}} 5 \underset{3}{\stackrel{?}{\rightarrow}} 5 \underset{1}{\stackrel{?}{\rightarrow}} \quad 3 \quad \rightarrow \quad 0 \\
& n=4 \quad 4 \underset{1}{\stackrel{C K}{\rightarrow}} 9 \underset{2}{\stackrel{W}{\rightarrow}} 10 \underset{2}{\stackrel{?}{\rightarrow}} 9 \underset{1}{\rightarrow} \quad 4 \rightarrow 0 \\
& n=5 \quad 5 \underset{1}{\stackrel{C K}{\rightarrow}} 14 \underset{2}{\stackrel{W}{\rightarrow}} \quad 35 \underset{1}{\stackrel{C B}{\rightarrow}} \quad 35 \underset{2}{\rightarrow \rightarrow} \quad 14 \underset{1}{\rightarrow} \quad 5 \quad \rightarrow 0
\end{aligned}
$$

For example, we have the Euler-Poincaré characteristic: $r k_{D}(M)=5-14+35-35+14-5=0$.

Proceeding exactly as before, we obtain for example when $n=4$ the following commutative diagram where all the rows are exact and the columns are exact but eventually the left one: 


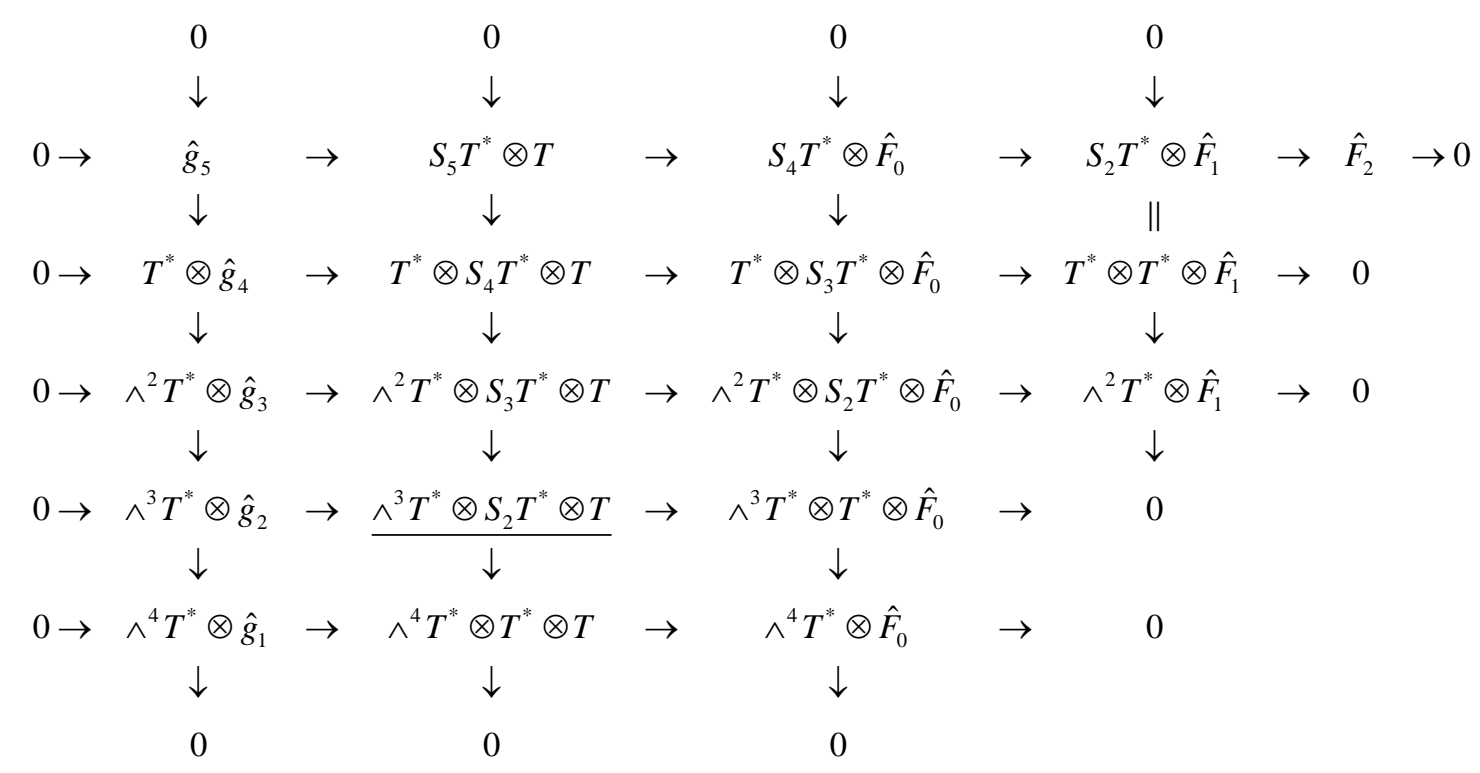

Nevertheless, the same (but very tricky now!) chase as before allows to prove that the bottom $\delta$-map in the first column on the left is again ... an epimorphism, a crucial result indeed, left again as a difficult exercise of diagram chasing (Hint: double step circular chase as before!).

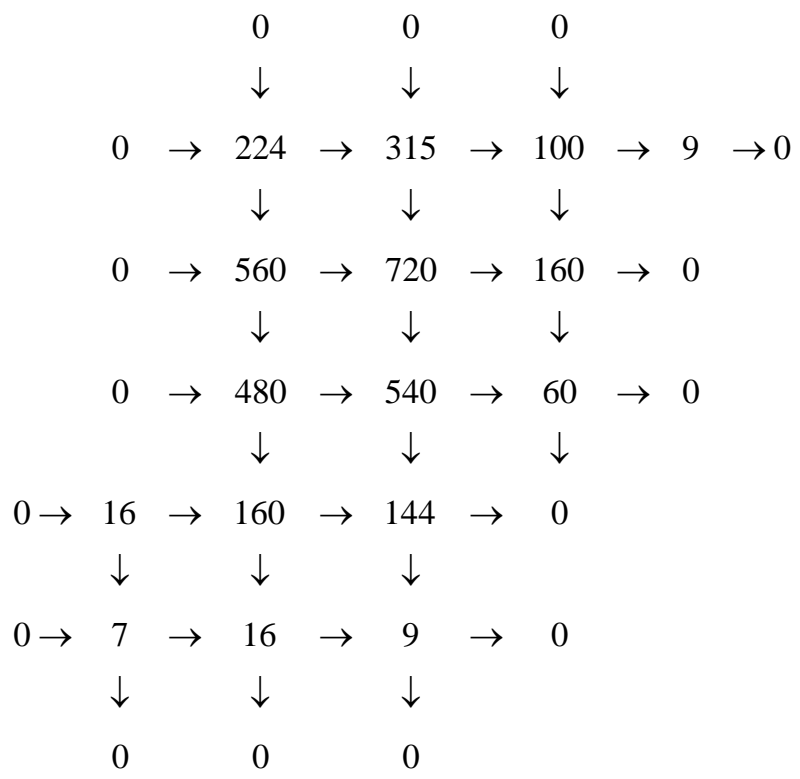

Of course, in view of the dimensions of the matrices involved (up to $540 \times 720$ ), we wish good luck to anybody trying to use computer algebra and refer to the computations done in ([1]) that have been done while knowing " $a$ priori " the dimensions that should be found.

Remark 4.1: Using the splitting of the Riemann tensor between the Ricci tensor and the Weyl tensor for the second column while taking into account the fact that the extension modules are torsion modules and thus that each component of the Weyl tensor is differentially dependent on the Ricci tensor, we obtain the following commutative and exact diagram: 


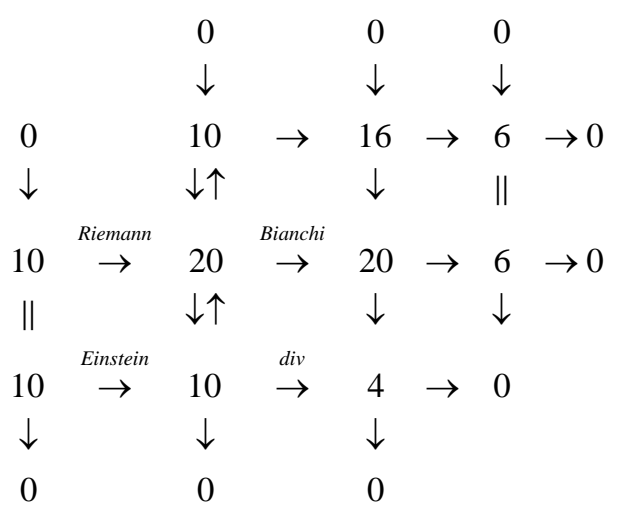

It follows that the 10 components of the Weyl tensor must satisfy a first order linear system with 16 equations, having 6 generating first order CC. The differential rank of the corresponding operator is thus equal to $16-6=10$ and such an operator defines a torsion module in which we have to look separately for each component of the Weyl tensor in order to prove that it is killed by the $\mathrm{Da}$ lembert operator ([16]). The situation is similar to that of the Cauchy-Riemann equations obtained when $n=2$ by considering the conformal Killing operator $C K$. Indeed, any complex transformation $y=f(x)$ must be solution of the (linear) first order system $y_{2}^{2}-y_{1}^{1}=0, y_{2}^{1}+y_{1}^{2}=0$ of finite Lie equations though we obtain $y_{11}^{1}+y_{22}^{1}=0, y_{11}^{2}+y_{22}^{2}=0$, that is $y^{1}$ and $y^{2}$ are separately killed by the second order Laplace operator $\Delta=d_{11}+d_{22}$. We obtain the following striking technical lemma explaining the so-called gauging procedure of the Lanczos potential.

Lemma 4.2: When $n=4$, the central vertical arrow $20 \rightarrow 4$ is just described by the contraction formula $\wedge^{2} T^{*} \otimes T^{*} \rightarrow \wedge^{2} T^{*} \otimes T \rightarrow T^{*}$ depending on the metric:

$$
L_{i j, k} \rightarrow L_{i}=\omega^{j k} L_{i j, k}
$$

Proof: Let us write down the Bianchi operator in the form:

$$
\nabla_{r} R_{l, i j}^{k}+\nabla_{i} R_{l, j r}^{k}+\nabla_{j} R_{l, r i}^{k}=B_{l, i j r}^{k}
$$

Contracting with $k=j=s$, we obtain:

$$
\nabla_{r} R_{l, i s}^{s}+\nabla_{i} R_{l, s r}^{s}+\nabla_{s} R_{l, r i}^{s}=B_{l, i s r}^{s}
$$

Setting as usual $R_{l, s r}^{s}=R_{l r}=R_{r l}$ with $\omega^{i j} R_{i j}=R$ and contracting with $\omega^{l i}$, we finally get :

$$
2 \nabla_{s} R_{r}^{s}-\nabla_{r} R=\omega^{i j} B_{i, j r s}^{s}
$$

as the way to use a contraction in order to exhibit Einstein equations.

With $n=4$, let us write down all the terms, using the Euclidean metric for simplicity instead of the Minkowski metric, recalling that only this later choice allows to find out both the Poincare group and the differential sequence with successive operators $K, R, B$ according to ([22]):

$$
B_{1,1 r s}^{s}+B_{2,2 r s}^{s}+B_{3,3 r s}^{s}+B_{4,4, r s}^{s}=C_{r}
$$


that is to say with all the terms:

$$
\begin{aligned}
& B_{1,1 r 1}^{1}+B_{1,1 r 2}^{2}+B_{1,1, r 3}^{3}+B_{1,1 r 4}^{4}+B_{2,2 r 1}^{1}+B_{2,2 r 2}^{2}+B_{2,2, r 3}^{3}+B_{2,2 r 4}^{4} \\
& +B_{3,3 r 1}^{1}+B_{3,3 r 2}^{2}+B_{3,3, r 3}^{3}+B_{3,3 r 4}^{4}+B_{4,4 r 1}^{1}+B_{4,4 r 2}^{2}+B_{4,4 r 3}^{3}+B_{4,4 r 4}^{4}=C_{r}
\end{aligned}
$$

where, in any case, we have $B_{1,1 r 1}^{1}=B_{2, r 2}^{2}=B_{3, r 3}^{3}=B_{4, r 4}^{4}=0$.

If we set $r=1$, the first line disappears because of the 3 -form $\wedge^{3} T^{*}$ and we are left with:

$$
B_{2,213}^{3}+B_{2,214}^{4}+B_{3,312}^{2}+B_{3,3,14}^{4}+B_{4,412}^{2}+B_{4,413}^{3}=C_{1}
$$

Using Hodge duality, we get with new indices:

$$
-B_{2,4}^{3}+B_{2,3}^{4}+B_{3,4}^{2}-B_{3,2}^{4}-B_{4,3}^{2}+B_{4,2}^{3}=C_{1}
$$

arriving finally to the formula:

$$
2\left(B_{3,4}^{2}+B_{4,2}^{3}+B_{2,3}^{4}\right)=C_{1}
$$

that is exactly twice the trace of the Lanczos tensor, namely:

$$
L_{12}^{2}+L_{13}^{3}+L_{14}^{4}=L_{1 r}^{r}
$$

This result explains why the Lanczos tensor $L_{i j, k}=-L_{j i, k}$ with 24 components is first reduced to 20 components through the condition $L_{i j, k}+L_{j k, i}+L_{k, i j}=0$ and finally to 16 components as in the diagram through the kernel of the above trace condition. It is thus impossible to understand this result, even for $n=4$, without the Spencer $\delta$-cohomology and absolutely impossible to generalize this result in arbitrary dimension without the combination of the $\delta$-cohomology and double duality in differential homological algebra.

Q.E.D.

Finally, using the previous definition $\operatorname{ad}(E)=\wedge^{n} T^{*} \otimes E^{*}$, such a result explains the confusion done by Lanczos and followers between the Riemann candidate $F_{2}$ or the Weyl candidate $\hat{F}_{2}$ and their respective formal adjoint vector bundles having of course the same fiber dimension but quite different transition rules under changes of local coordinates.

We notice that the changes of the successive orders are totally unusual and refer to ([22]) for more details on the computer algebra methods. In particular, when $n=4$, the conformal analogue of the Bianchi operator is now of order 2 , a result explaining why Lanczos and followers never succeeded adapting the Lanczos tensor potential $L$ for the Weyl operator. We understand therefore that the solution of what we called "Lanczos secret" must be depending on a quite different homological framework. It is only after exhibiting it in the last section below that we will be able to say that we have thus solved the Riemann-Lanczos and Weyl-Lanczos parametrization problems in arbitrary dimension. In the meantime, we provide two examples that can be fully computed as a way to understand the use of adjoint differential sequences.

\section{Motivating Examples}

The two following examples will show how the differential extension modules 
may depend on the Vessiot structure constants.

Example 5.1: With $m=n=2, q=1, K=\mathbb{Q}\langle\omega\rangle$ and $\omega=(\alpha, \beta)$ with $\alpha \in T^{*}, \beta \in \wedge^{2} T^{*}$, let us consider the Lie operator $\mathcal{D}: T \rightarrow \Omega: \xi \rightarrow \mathcal{L}(\xi) \omega=(A=\mathcal{L}(\xi) \alpha, B=\mathcal{L}(\xi) \beta)$. The corresponding first order system:

$$
A_{i} \equiv \alpha_{r} \partial_{i} \xi^{r}+\xi^{r} \partial_{r} \alpha_{i}=0, B \equiv \beta \partial_{r} \xi^{r}+\xi^{r} \partial_{r} \beta=0
$$

is involutive whenever $\beta \neq 0$ and $d \alpha=c \beta$ where now $d$ is the standard exterior derivative and $c=c s t$, exactly as in ([19], p 438-440). We have the differential sequence:

$$
0 \rightarrow \Theta \rightarrow T \stackrel{\mathcal{D}}{\rightarrow} T^{*} \times_{X} \wedge^{2} T^{*} \stackrel{\mathcal{D}_{1}}{\rightarrow} \wedge^{2} T^{*} \rightarrow 0
$$

with $\xi \rightarrow\left(A_{1}, A_{2}, B\right) \rightarrow C \rightarrow 0$ or the resolution:

$$
0 \rightarrow D \stackrel{\mathcal{D}_{1}}{\rightarrow} D^{3} \stackrel{\mathcal{D}}{\rightarrow} D^{2} \stackrel{p}{\rightarrow} M \rightarrow 0
$$

Multiplying $\left(A_{1}, A_{2}, B\right)$ respectively by $\left(\mu^{1}, \mu^{2}, \mu^{3}\right)$, we obtain $\operatorname{ad}(\mathcal{D})$ in the form:

$$
\begin{aligned}
& -\alpha_{1}\left(\partial_{1} \mu^{1}+\partial_{2} \mu^{2}\right)-\beta\left(\partial_{1} \mu^{3}-c \mu^{2}\right)=v^{1}, \\
& -\alpha_{2}\left(\partial_{1} \mu^{1}+\partial_{2} \mu^{2}\right)-\beta\left(\partial_{2} \mu^{3}+c \mu^{1}\right)=v^{2}
\end{aligned}
$$

Then, multiplying $\partial_{1} A_{2}-\partial_{2} A_{1}-c B=C$ by $\lambda$, we obtain $\operatorname{ad}\left(\mathcal{D}_{1}\right)$ as:

$$
\partial_{2} \lambda=\mu^{1},-\partial_{1} \lambda=\mu^{2},-c \lambda=\mu^{3}
$$

We have therefore to consider the two cases:

- $c=0$ : We have the new CC $\partial_{1} \mu^{1}+\partial_{2} \mu^{2}=0$ and $\mu^{3}=0$. It follows that the torsion module $\operatorname{ext}^{1}(M) \neq 0$ is generated by the residue of $\mu^{3}=v^{\prime}$ because $\alpha \neq 0$ and we may thus suppose that $\alpha_{1} \neq 0$. As for $\operatorname{ext}^{2}(M)$, this torsion module is just defined by the system $\partial_{2} \lambda=0, \partial_{1} \lambda=0$ for $\lambda$ and thus $\operatorname{ext}^{2}(M) \neq 0$.

- $c \neq 0$ : We must have the new CC:

$$
\partial_{1} \mu^{3}-c \mu^{2}=0, \partial_{2} \mu^{3}+c \mu^{1}=0 \Rightarrow \partial_{1} \mu^{1}+\partial_{2} \mu^{2}=0
$$

It follows that $\operatorname{ext}^{1}(M)$ is now generated by the residue of $\partial_{1} \mu^{1}+\partial_{2} \mu^{2}=v^{\prime}$. Finally, $\operatorname{ker}\left(\operatorname{ad}\left(\mathcal{D}_{1}\right)\right)$ is defined by $\lambda=0$ and thus $\operatorname{ext}^{2}(M)=0$.

Hence, both ext ${ }^{1}(M)$ and ext $^{2}(M)$ highly depend on the Vessiot structure constant $c$.

\section{Example 5.2: (Contact transformations)}

With $m=n=3, q=1, K=\mathbb{Q}\left(x^{1}, x^{2}, x^{3}\right)$ or simply $\mathbb{Q}(x)$, we may introduce the 1-form $\alpha=d x^{1}-x^{3} d x^{2} \in T^{*}$ and consider the system of finite Lie equations defined by $j_{1}(f)^{-1}(\alpha)=\rho(x) \alpha$. Eliminating the factor $\rho$ and linearizing at the $q$-jet of the identity, we obtain the first order involutive system of infinitesimal Lie equations:

$$
\left\{\begin{array}{l}
\eta^{3} \equiv \partial_{3} \xi^{3}+\partial_{2} \xi^{2}-\partial_{1} \xi^{1}+2 x^{3} \partial_{1} \xi^{2}=0 \\
\eta^{2} \equiv \partial_{3} \xi^{1}-x^{3} \partial_{3} \xi^{2}=0 \\
\eta^{1} \equiv \partial_{2} \xi^{1}-x^{3} \partial_{2} \xi^{2}+x^{3} \partial_{1} \xi^{1}-\left(x^{3}\right)^{2} \partial_{1} \xi^{2}-\xi^{3}=0
\end{array}\right.
$$


with two equations of class 3 , one equation of class 2 and thus one CC of order 1 , namely $d_{3} \eta^{1}-d_{2} \eta^{2}-x^{3} d_{1} \eta^{2}+\eta^{3}=0$. Now, it is well known that this contact operator $\mathcal{D}=\mathcal{D}_{0}$ can be parametrized by an operator $\mathcal{D}_{-1}$ as follows:

$$
-x^{3} \partial_{3} \phi+\phi=\xi^{1},-\partial_{3} \phi=\xi^{2}, \partial_{2} \phi+x^{3} \partial_{1} \phi=\xi^{3} \Rightarrow \xi^{1}-x^{3} \xi^{2}=\phi
$$

and thus $M \simeq D$. We have obtained the following formally exact sequence which is nevertheless not strictly exact because $\mathcal{D}_{-1}$ is not formally integrable:

$$
\begin{gathered}
0 \rightarrow \phi \stackrel{\mathcal{D}_{-1}}{\rightarrow} \xi \stackrel{\mathcal{D}}{\rightarrow} \eta \stackrel{\mathcal{D}_{1}}{\rightarrow} \zeta \rightarrow 0 \\
0 \rightarrow 1 \rightarrow 3 \rightarrow 3 \rightarrow 1 \rightarrow 0 \Leftrightarrow 0 \rightarrow D \rightarrow D^{3} \rightarrow D^{3} \rightarrow D \rightarrow 0
\end{gathered}
$$

As $M$ is therefore free and thus projective, it follows that the adjoint sequence is exact too.

Coming back to the Vessiot structure equations, we notice that $\alpha$ is not invariant by the contact Lie pseudogroup and cannot be considered as an associated geometric object. We have shown in ([9], p 684-691) that the corresponding geometric object is a 1-form density $\omega$ leading to the system of infinitesimal Lie equations in Medolaghi form:

$$
\Omega_{i} \equiv(\mathcal{L}(\xi) \omega)_{i} \equiv \omega_{r} \partial_{i} \xi^{r}-\frac{1}{2} \omega_{i} \partial_{r} \xi^{r}+\xi^{r} \partial_{r} \omega_{i}=0
$$

and to the only Vessiot structure equation:

$$
\omega_{1}\left(\partial_{2} \omega_{3}-\partial_{3} \omega_{2}\right)+\omega_{2}\left(\partial_{3} \omega_{1}-\partial_{1} \omega_{3}\right)+\omega_{3}\left(\partial_{1} \omega_{2}-\partial_{2} \omega_{3}\right)=c
$$

with the only structure constant $c$. In the present contact situation, we may choose $\omega=\left(1,-x^{3}, 0\right)$ and get $c=1$ but we may also choose $\omega=(1,0,0)$ and get $c=0$, these two choices both bringing an involutive system. Let us prove that the situation becomes completely different with the new system:

$$
-2 \Omega_{1} \equiv \partial_{3} \xi^{3}+\partial_{2} \xi^{2}-\partial_{1} \xi^{1}=0, \Omega_{2} \equiv \partial_{2} \xi^{1}=0, \Omega_{3} \equiv \partial_{3} \xi^{1}=0
$$

having the only CC $d_{2} \Omega_{3}-d_{3} \Omega_{2}=0$.

Multiplying the three previous equations by the three test functions $\mu$, the only CC by the test function $\lambda$ and integrating by parts, we get the adjoint operators:

$$
\begin{gathered}
0=\mu^{1}, \partial_{3} \lambda=\mu^{2},-\partial_{2} \lambda=\mu^{3} \\
\partial_{1} \mu^{1}-\partial_{2} \mu^{2}-\partial_{3} \mu^{3}=v^{1},-\partial_{2} \mu^{1}=v^{2},-\partial_{3} \mu^{1}=v^{3}
\end{gathered}
$$

It follows that $0 \neq D \xi^{1}=t(M) \subset M$ with a strict inclusion and $\operatorname{ext}^{1}(M) \neq 0$. Similarly, $\operatorname{ker}\left(\operatorname{ad}\left(\mathcal{D}_{1}\right)\right)$ is defined by $\partial_{2} \lambda=0, \partial_{3} \lambda=0$ and thus $\operatorname{ext}^{2}(M) \neq 0$.

Our problem will be now to construct and compare the differential sequences:

$$
\begin{gathered}
\stackrel{\mathcal{D}_{-1}}{\rightarrow} \xi \stackrel{\mathcal{D}}{\rightarrow} \Omega \stackrel{\mathcal{D}_{1}}{\rightarrow} C \\
\operatorname{ad}\left(\mathcal{D}_{-1}\right) \operatorname{ad}(\mathcal{D}) \quad \operatorname{ad}\left(\mathcal{D}_{1}\right) \\
\theta \leftarrow \mu
\end{gathered}
$$

For this, linearizing the only Vessiot structure equation, we get the CC operator $\mathcal{D}_{1}$ and the corresponding system $\mathcal{D}_{1} \Omega=0$ in the form: 


$$
\begin{aligned}
& \omega_{1}\left(\partial_{2} \Omega_{3}-\partial_{3} \Omega_{2}\right)+\omega_{2}\left(\partial_{3} \Omega_{1}-\partial_{1} \Omega_{3}\right)+\omega_{3}\left(\partial_{1} \Omega_{2}-\partial_{2} \Omega_{1}\right) \\
& +\left(\partial_{2} \omega_{3}-\partial_{3} \omega_{2}\right) \Omega_{1}+\left(\partial_{3} \omega_{1}-\partial_{1} \omega_{3}\right) \Omega_{2}+\left(\partial_{1} \omega_{2}-\partial_{2} \omega_{3}\right) \Omega_{3}=0
\end{aligned}
$$

Multiplying on the left by the test function $\lambda$ and integrating by parts, we get the operator $\operatorname{ad}\left(\mathcal{D}_{1}\right)$ in the form:

$$
\left\{\begin{array}{l}
\Omega_{1} \rightarrow \omega_{3} \partial_{2} \lambda-\omega_{2} \partial_{3} \lambda+2\left(\partial_{2} \omega_{3}-\partial_{3} \omega_{2}\right) \lambda=\mu^{1} \\
\Omega_{2} \rightarrow \omega_{1} \partial_{3} \lambda-\omega_{3} \partial_{1} \lambda+2\left(\partial_{3} \omega_{1}-\partial_{1} \omega_{3}\right) \lambda=\mu^{2} \\
\Omega_{3} \rightarrow \omega_{2} \partial_{1} \lambda-\omega_{1} \partial_{2} \lambda+2\left(\partial_{1} \omega_{2}-\partial_{2} \omega_{1}\right) \lambda=\mu^{3}
\end{array}\right.
$$

We obtain therefore the crucial formula $2 c \lambda=\omega_{i} \mu^{i}$ showing how the previous sequences are essentially depending on the Vessiot structure constant $c$. Indeed, if $c \neq 0$, then $\mu=0 \Rightarrow \lambda=0$ and the operator $\operatorname{ad}\left(\mathcal{D}_{1}\right)$ is injective. This is the case when $\omega=\left(1,-x^{3}, 0\right) \Rightarrow c=1 \Rightarrow \lambda=0$. On the contrary, if $c=0$, then the operator $\operatorname{ad}\left(\mathcal{D}_{1}\right)$ may not be injective as can be seen by choosing $\omega=(1,0,0)$. Indeed, in this case we get a kernel defined by $\partial_{3} \lambda=0, \partial_{2} \lambda=0$.

We invite the reader to treat similarly the case of unimodular contact transformations, namely transformations preserving the 1-form $\alpha=d x^{1}-x^{3} d x^{2}$, thus also the 2-form $\beta=d \alpha=d x^{2} \wedge d x^{3}$ and even the 3-form $d x^{1} \wedge d x^{2} \wedge d x^{3}$ that can be used as a volume form. The Vessiot structure equations for the ground geometric object $\omega=(\alpha, \beta)$ are now $d \alpha=c^{\prime} \beta, d \beta=c^{\prime \prime} \alpha \wedge \beta$ with the only striking Jacobi condition $c^{\prime} c^{\prime \prime}=0$ (See [1] for more details).

\section{Generalized Lanczos Problem}

In this last section, we prove that the following theorem allows to solve locally the Lanczos problem in a similar way for any Lie group of transformations:

Theorem 6.1: The Spencer sequence for any Lie operator $\mathcal{D}$ which is coming from a Lie group of transformations, with a Lie group $G$ acting on $X$, is (locally) isomorphic to the tensor product of the Poincaré sequence for the exterior derivative by the Lie algebra $\mathcal{G}$ of $G$.

Proof: If $M$ is the differential module defined by $\mathcal{D}$, we want to prove that the extension modules ext ${ }^{1}(M)$ and ext $^{2}(M)$ vanish, that is, if $\mathcal{D}_{1}$ generates the CC of $\mathcal{D}$ but also $\mathcal{D}_{2}$ generates the CC of $\mathcal{D}_{1}$, then $\operatorname{ad}(\mathcal{D})$ generates the CC of $\operatorname{ad}\left(\mathcal{D}_{1}\right)$ and $\operatorname{ad}\left(\mathcal{D}_{1}\right)$ generates the CC of $\operatorname{ad}\left(\mathcal{D}_{2}\right)$. We also remind the reader that we have shown in ([18] [22]) that it is not easy to exhibit the CC of the Maxwell or Morera parametrizations when $n=3$ and that a direct checking for $n=4$ should be strictly impossible. It has been proved by L. P. Eisenhart in 1926 ([20]) that the solution space $\Theta$ of the Killing system has $n(n+1) / 2$ infinitesimal generators $\left\{\theta_{\tau}\right\}$ linearly independent over the constants if and only if $\omega$ had constant Riemannian curvature, namely zero in our case. As we have a transitive Lie group of transformations preserving the metric considered as a transitive Lie pseudogroup, the three classical theorems of Sophus Lie assert than $\left[\theta_{\rho}, \theta_{\sigma}\right]=c_{\rho \sigma}^{\tau} \theta_{\tau}$ where the structure constants $c$ define a Lie algebra $\mathcal{G}$. We have therefore $\xi \in \Theta \Leftrightarrow \xi=\left\{\lambda^{\tau} \theta_{\tau} \mid \lambda^{\tau}=c s t\right\}$. Hence, we 
may replace locally the Killing system by the system $\partial_{i} \lambda^{\tau}(x)=0$, getting therefore the differential sequence:

$$
0 \rightarrow \Theta \rightarrow \wedge^{0} T^{*} \otimes G \stackrel{d}{\rightarrow} \wedge^{1} T^{*} \otimes G \stackrel{d}{\rightarrow} \cdots \stackrel{d}{\rightarrow} \wedge^{n} T^{*} \otimes G \rightarrow 0
$$

which is the tensor product of the Poincare sequence by $\mathcal{G}$. Finally, it follows from the above Theorem that the extension modules considered do not depend on the resolution used and thus vanish because the Poincare sequence is self adjoint (up to sign), that is $\operatorname{ad}(d)$ generates the CC of $a d(d)$ at any position, exactly like $d$ generates the CC of $d$ at any position. This (difficult) result explains why the adjoint differential modules we shall meet will be torsion-free or even reflexive. We invite the reader to compare with the situation of the Maxwell equations in electromagnetisme (See ([12], p 492-494) for more details). However, we have explained in ([11] [13]) why neither the Janet sequence nor the Poincaré sequence can be used in physics and must be replaced by the Spencer sequence which is another resolution of $\Theta$. Though this is out of the scope of this paper, we shall nevertheless shortly describe the relation existing between the above results and the Spencer operator, thus the Spencer sequence. For this, let us define for any $q \geq 0$ the section $\xi_{q}=\lambda^{\tau}(x) j_{q}\left(\theta_{\tau}\right)(x)=\left(\xi_{\mu}^{k}(x)=\lambda^{\tau}(x) \partial_{\mu} \theta_{\tau}^{k}(x)\right) \in R_{q}$. With the standard notations of ([8] [11] [12]) and $0 \leq|\mu| \leq q$, the components of the Spencer operator become:

$$
\begin{aligned}
& \xi_{q+1} \in R_{q+1} \rightarrow j_{1}\left(\xi_{q}\right)-\xi_{q+1}=\left(\partial_{i} \xi_{\mu}^{k}(x)-\xi_{\mu+1_{i}}^{k}(x)\right) \\
& =\left(\left(\partial_{i} \lambda^{\tau}(x)\right) \partial_{\mu} \theta_{\tau}^{k}(x) \xi\right) \in T^{*} \otimes R_{q}
\end{aligned}
$$

when $q$ is large enough, that is $q=2$ for the Killing system and $q=3$ for the conformal Killing system in arbitrary dimension ([43]), we have involutive systems with vanishing symbols because both are finite-type. We obtain therefore the desired identification justifying our claim.

Q.E.D.

Corollary 6.2: When $\mathcal{D}$ is the Killing operator or the conformal Killing operator, then $\operatorname{ext}^{1}(M)=0$, ext $t^{2}(M)=0$ and there is no gap. Moreover, if the differential module $M$ defined by $\mathcal{D}$ is a torsion module as in the Theorem, then we have $\operatorname{ext}^{0}(M)=\operatorname{hom}_{D}(M, D)=0$ in any case.

\section{Conclusion}

E. Vessiot discovered the so-called Vessiot structure equations as early as in 1903 and, only a few years later, E. Cartan discovered the so-called Maurer-Cartan structure equations. Both are depending on a certain number of constants like the single geometric structure constant of the constant Riemannian curvature for the first and the many algebraic structure constants of Lie algebra for the second. However, Cartan and followers never acknowledged the existence of another approach which is therefore still totally ignored today, in particular by physicists. Now, it is well known that the structure constants of a Lie algebra play a fundamental part in the Chevalley-Eilenberg cohomology of Lie algebras and their 
deformation theory. It was thus a challenge to associate the Vessiot structure constants with other homological properties related to systems of Lie equations, namely the extension modules determined by Lie operators. As a striking consequence, such a possibility opens a new way to understand and revisit the various contradictory works done during the last fifty years or so by different groups of researchers, using respectively Cartan, Gröbner or Janet bases while looking for a modern interpretation of the work done by C. Lanczos from 1938 to 1962. However, the reader must not forget that the Weyl tensor was not known by Lanczos, even as late as in 1967, and that it was not possible to discover any solution of the parametrization problem by potentials through double duality before 1990/1995, that is too late for the many people already engaged in this type of research. We finally hope that this paper will open a new domain for applying computer algebra while offering a collection of useful test examples.

\section{Conflicts of Interest}

The author declares no conflicts of interest regarding the publication of this paper.

\section{References $^{1}$}

[1] Pommaret, J.-F. (2016) Deformation Theory of Algebraic and Geometric Structures. Lambert Academic Publisher (LAP), Saarbrucken, Germany. In: Dubreil, S.P. and Malliavin, M.-P., Eds., Topics in Invariant Theory, Lambert Academic Publisher (LAP), Saarbrucken, Springer Lecture Notes in Mathematics, Vol. 1478, 244-254 https://arxiv.org/abs/1207.1964 https://doi.org/10.1007/BFb0083506

[2] Lanczos, C. (1938) Annals of Mathematics, 39, 842-850. https://doi.org/10.2307/1968467

[3] Lanczos, C. (1949) Reviews of Modern Physics, 21, 497-502. https://doi.org/10.1103/RevModPhys.21.497

[4] Lanczos, C. (1962) Reviews of Modern Physics, 34, 379-389. https://doi.org/10.1103/RevModPhys.34.379

[5] Lanczos, C. (1962) Annales Scientifiques de P Université de Clermont-Ferrand 2, Mathématiques, 8, 167-170. http://www.numdam.org

[6] O’Donnell, P. and Pye, H. (2010) EJTP, 24, 327-350.

[7] Pommaret, J.-F. (2019) Journal of Modern Physics, 10, 1566-1595. https://arxiv.org/abs/1802.02430 https://doi.org/10.4236/jmp.2019.1013104

[8] Pommaret, J.-F. (1978) Systems of Partial Differential Equations and Lie Pseudogroups. Gordon and Breach, New York; Russian Translation by MIR, Moscow, 1983.

[9] Pommaret, J.-F. (1983) Differential Galois Theory. Gordon and Breach, New York.

[10] Pommaret, J.-F. (1988) Lie Pseudogroups and Mechanics. Gordon and Breach, New York.

[11] Pommaret, J.-F. (1994) Partial Differential Equations and Group Theory: New Pers-

${ }^{1}$ We have only quoted the recent references on the Lanczos potential published after 2000. 
pectives for Applications (Mathematics and Its Applications 293). Wolters Kluwer, Alphen aan den Rijn. https://doi.org/10.1007/978-94-017-2539-2

[12] Pommaret, J.-F. (2001) Partial Differential Control Theory. Wolters Kluwer, Alphen aan den Rijn, 957 p. https://doi.org/10.1007/978-94-010-0854-9

[13] Pommaret, J.-F. (2018) New Mathematical Methods for Physics, Mathematical Physics Books. Nova Science Publishers, New York, 150 p.

[14] Pommaret, J.-F. (2013) Journal of Modern Physics, 4, 223-239. https://doi.org/10.4236/jmp.2013.48A022

[15] Pommaret, J.-F. (2016) Journal of Modern Physics, 7, 699-728. https://doi.org/10.4236/jmp.2016.77068

[16] Pommaret, J.-F. (2017) Journal of Modern Physics, 8, 2122-2158. https://doi.org/10.4236/jmp.2017.813130

[17] Pommaret, J.-F. (2019) Journal of Modern Physics, 10, 1454-1486. https://doi.org/10.4236/jmp.2019.1012097

[18] Pommaret, J.-F. (2021) Journal of Modern Physics, 12, 453-482. https://doi.org/10.4236/jmp.2021.124032

[19] Vessiot, E. (1903) Annales scientifiques de I École Normale Supérieure, 20, 411-451. https://doi.org/10.24033/asens.529

[20] Eisenhart, L.P. (1926) Riemannian Geometry. Princeton University Press, Princeton. https://doi.org/10.1090/coll/008

[21] Pommaret, J.-F. (2019) Journal of Modern Physics, 10, 371-401. https://doi.org/10.4236/jmp.2019.103025

[22] Pommaret, J.-F. (2020) Journal of Modern Physics, 11, 1672-1710. https://doi.org/10.4236/jmp.2020.1110104

[23] Pommaret, J.-F. (2012) Spencer Operator and Applications: From Continuum Mechanics to Mathematical Physics. In: Gan, Y., Ed., Continuum Mechanics-Progress in Fundamentals and Engineering Applications, InTech, Rijeka, Chapter 1. https://www.intechopen.com/books/continuum-mechanics-progress-in-fundament als-and-engineering-applications

[24] Goldschmidt, H. (1968) Annales scientifiques de PÉcole Normale Supérieure, Série 4, 1, 617-625. https://doi.org/10.24033/asens.1173

[25] Janet, M. (1920) Journal de Mathematiques, 8, 65-151.

[26] Spencer, D.C. (1965) Bulletin of the American Mathematical Society, 75, 1-114.

[27] Kunz, E. (1985) Introduction to Commutative Algebra and Algebraic Geometry. Birkhäuser, Boston. https://doi.org/10.1007/978-1-4612-5290-0

[28] Rotman, J.J. (1979) An Introduction to Homological Algebra. Academic Press, Cambridge.

[29] Kashiwara, M. (1995) Algebraic Study of Systems of Partial Differential Equations. Mémoires de la Société Mathématique de France 63, Transl. from Japanese of His 1970 Master's Thesis.

[30] Oberst, U. (1990) Acta Applicandae Mathematicae, 20, 1-175. https://doi.org/10.1007/BF00046908

[31] Schneiders, J.-P. (1994) Bulletin de la Société Royale des Sciences de Liège, 63, 223-295.

[32] Pommaret, J.-F. (1995) Comptes Rendus de I Académie des Sciences Paris, Série I, 320, 1225-1230. 
[33] Zerz, E. (2000) Topics in Multidimensional Linear Systems Theory. Lecture Notes in Control and Information Sciences 256, Springer, Berlin.

[34] Pommaret, J.-F. (2005) Algebraic Analysis of Control Systems Defined by Partial Differential Equations. In: Advanced Topics in Control Systems Theory, Lecture Notes in Control and Information Sciences 311, Chapter 5, Springer, Berlin, 155223. https://doi.org/10.1007/11334774 5

[35] Pommaret, J.-F. and Quadrat, A. (1999) Systems \& Control Letters, 37, 247-260. https://doi.org/10.1016/S0167-6911(99)00030-4

[36] Pommaret, J.-F. (2010) Acta Mechanica, 215, 43-55. https://doi.org/10.1007/s00707-010-0292-y

[37] Edgar, S.B. and Höglund, A. (2000) General Relativity and Gravitation, 32, 23072318. https://doi.org/10.1023/A:1001951609641 http://rspa.royalsocietypublishing.org/content/royprsa/453/1959/835.full.pdf

[38] Dolan, P. and Gerber, A. (2002) Janet-Riquier Theory and the Riemann-Lanczos Problem in 2 and 3 Dimensions.

[39] Edgar, S.B. (2003) Journal of Mathematical Physics, 44, 5375-5385. https://arxiv.org/abs/gr-qc/0302014 https://doi.org/10.1063/1.1619203

[40] Edgar, S.B. and Senovilla, J.M.M. (2004) Classical and Quantum Gravity, 21, L133. https://arxiv.org/abs/gr-qc/0408071 https://doi.org/10.1088/0264-9381/21/22/L01

[41] O’Donnell, P. (2004) General Reativity and Gravitation, 36, 1415-1422. https://doi.org/10.1023/B:GERG.0000022577.11259.e0

[42] Pommaret, J.-F. (2013) Multidimensional Systems and Signal Processing, 26, 405437. https://doi.org/10.1007/s11045-013-0265-0

[43] Pommaret, J.-F. (2020) The Conformal Group Revisited. https://arxiv.org/abs/2006.03449 